\title{
Republic of Equatorial Guinea: Statistical Appendix
}

This Statistical Appendix for the Republic of Equatorial Guinea was prepared by a staff team of the International Monetary Fund as background documentation for the periodic consultation with the member country. It is based on the information available at the time it was completed on May 22, 2007. The views expressed in this document are those of the staff team and do not necessarily reflect the views of the government of Germany or the Executive Board of the IMF.

The policy of publication of staff reports and other documents by the IMF allows for the deletion of market-sensitive information.

Copies of this report are available to the public from

International Monetary Fund • Publication Services

700 19th Street, N.W. • Washington, D.C. 20431

Telephone: (202) 623-7430 • Telefax: (202) 623-7201

E-mail: publications@imf.org • Internet: http://www.imf.org

Price: $\$ 18.00$ a copy

\section{International Monetary Fund} Washington, D.C. 



\section{INTERNATIONAL MONETARY FUND \\ REPUBLIC OF EQUATORIAL GUINEA}

\section{Statistical Appendix}

Prepared by a staff team consisting of Janet Stotsky (head), Helmut Franken, Plamen Iossifov (all AFR), and Lisbeth Rivas (STA)

Approved by the African Department

May 22, 2007

Contents

Page

Tables

1. GDP by Sector of Origin, 2001-06 (in current prices) ..........................................2

2. GDP by Sector of Origin, 2001-06 (in percent of GDP) .........................................

3. GDP by Sector of Origin, 2001-06 (in constant prices) .........................................4

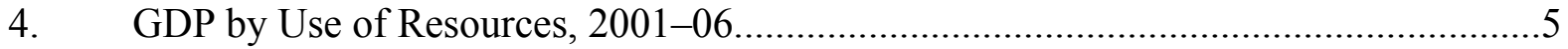

5. Consumer Price Index, 2001-06 ......................................................................6

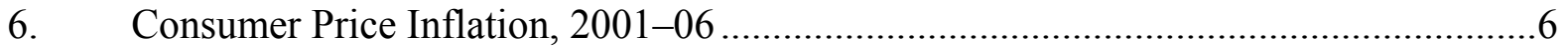

7. Summary of Central Government Financial Operation, 2001-06 .............................. 7

8. Detailed Central Government Revenue, 2001-06 ..................................................8

9. Detailed Central Government Expenditure and Financing, 2001-06 ..........................9

10. Fiscal Indicators, 2001-06 .............................................................................. 10

11. Public Investment Program, 2004-06 (Execution) and 2007-08 (Budgeted) ............11

12. Monetary Survey, 2001-06............................................................................ 12

13. Central Bank and Commercial Banks, 2001-06 .....................................................13

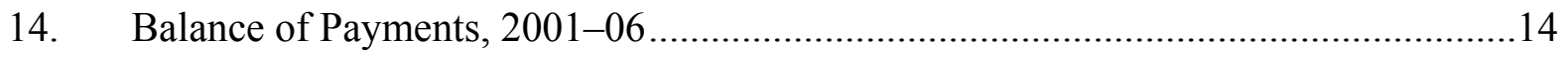

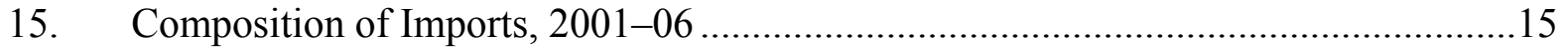

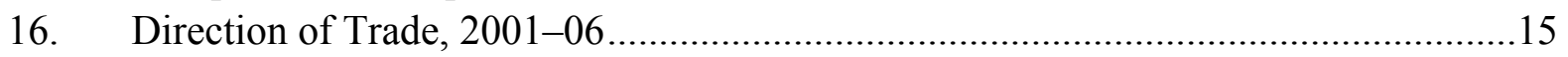

17. Scheduled External Public Debt Service, 2001-06 ...............................................16

18. External Medium- and Long-Term Outstanding Public Debt, 2001-06 ....................16

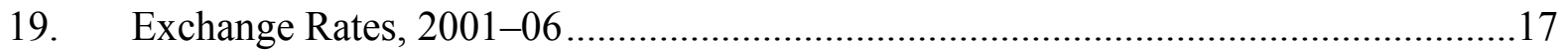

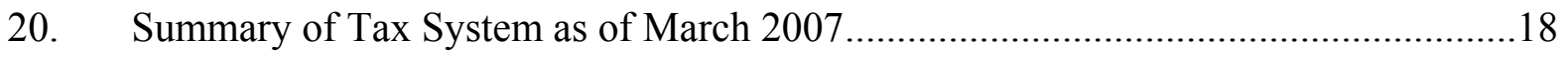


Table 1. Equatorial Guinea: GDP by Sector of Origin, 2001-06

\begin{tabular}{|c|c|c|c|c|c|c|}
\hline & 2001 & 2002 & 2003 & 2004 & 2005 & $\begin{array}{r}2006 \\
\text { Est. }\end{array}$ \\
\hline & \multicolumn{6}{|c|}{ (Billions of current CFA francs) } \\
\hline Primary sector & $1,076.8$ & $1,246.6$ & $1,413.3$ & $2,196.2$ & $3,366.0$ & $3,697.9$ \\
\hline Non-oil & 84.9 & 94.1 & 97.1 & 104.5 & 112.3 & 120.9 \\
\hline Agriculture & 49.9 & 57.3 & 60.0 & 67.7 & 73.9 & 81.8 \\
\hline Forestry & 33.5 & 34.7 & 34.7 & 34.4 & 35.7 & 36.3 \\
\hline Fishing & 1.4 & 2.1 & 2.3 & 2.5 & 2.6 & 2.8 \\
\hline Oil & 991.9 & $1,152.5$ & $1,316.2$ & $2,091.7$ & $3,253.7$ & $3,577.0$ \\
\hline Secondary sector & 121.9 & 164.8 & 193.0 & 265.0 & 452.1 & 612.0 \\
\hline Manufacturing & 73.9 & 94.6 & 109.9 & 152.1 & 301.4 & 381.4 \\
\hline Oil derivatives & 71.4 & 91.7 & 106.3 & 147.5 & 295.7 & 374.6 \\
\hline Other manufacturing & 2.5 & 2.9 & 3.6 & 4.6 & 5.7 & 6.7 \\
\hline Electricity & 5.8 & 7.2 & 8.4 & 12.4 & 17.3 & 24.4 \\
\hline Construction & 42.1 & 63.0 & 74.7 & 100.5 & 133.4 & 206.2 \\
\hline Tertiary sector & 53.3 & 74.7 & 86.7 & 96.0 & 115.7 & 134.1 \\
\hline Trade and commerce & 18.9 & 26.0 & 27.6 & 31.2 & 34.4 & 39.7 \\
\hline Transport and communications & 2.6 & 4.3 & 4.6 & 5.2 & 5.7 & 6.7 \\
\hline Finance and housing & 3.8 & 4.4 & 4.7 & 7.2 & 12.6 & 16.0 \\
\hline Public administration & 19.4 & 30.0 & 34.5 & 34.9 & 43.8 & 47.6 \\
\hline Other services & 8.5 & 9.9 & 15.4 & 17.5 & 19.2 & 21.7 \\
\hline GDP at factor costs & $1,252.0$ & $1,486.1$ & $1,693.1$ & $2,557.1$ & $3,933.8$ & $4,443.9$ \\
\hline Of which: non-oil GDP & 260.1 & 333.6 & 376.8 & 465.5 & 680.1 & 867.0 \\
\hline Import duties and subsidies & 21.6 & 23.5 & 31.0 & 30.9 & 33.0 & 33.7 \\
\hline Discrepancy & 0.0 & 0.0 & 0.0 & 0.0 & 0.0 & 0.0 \\
\hline GDP at market prices & $1,273.6$ & $1,509.6$ & $1,724.1$ & $2,588.0$ & $3,966.8$ & $4,477.6$ \\
\hline \multirow[t]{2}{*}{ Of which: non-oil GDP } & 281.7 & 357.1 & 407.8 & 496.4 & 713.0 & 900.7 \\
\hline & \multicolumn{6}{|c|}{ (Annual percentage change) } \\
\hline Primary sector & 35.0 & 15.8 & 13.4 & 55.4 & 53.3 & 9.9 \\
\hline Non-oil primary sector & -1.1 & 10.8 & 3.2 & 7.6 & 7.5 & 7.7 \\
\hline Agriculture & 4.0 & 14.7 & 4.7 & 12.8 & 9.3 & 10.7 \\
\hline Forestry & -9.1 & 3.5 & 0.0 & -1.1 & 4.0 & 1.6 \\
\hline Fishing & 55.6 & 46.9 & 12.5 & 5.6 & 6.7 & 6.5 \\
\hline Oil sector & 39.4 & 16.2 & 14.2 & 58.9 & 55.6 & 9.9 \\
\hline Secondary sector & 207.8 & 35.2 & 17.1 & 37.3 & 70.6 & 35.4 \\
\hline Manufacturing & 494.7 & 28.0 & 16.2 & 38.4 & 98.1 & 26.5 \\
\hline Oil derivatives & 591.3 & 28.4 & 16.0 & 38.8 & 100.5 & 26.7 \\
\hline Other manufacturing & 19.3 & 17.4 & 23.8 & 26.9 & 23.4 & 18.0 \\
\hline Electricity & 43.1 & 23.3 & 16.7 & 47.1 & 39.8 & 41.0 \\
\hline Construction & 82.4 & 49.5 & 18.6 & 34.5 & 32.7 & 54.6 \\
\hline Tertiary sector & 43.6 & 40.2 & 16.0 & 10.7 & 20.6 & 15.9 \\
\hline Trade and commerce & 42.1 & 37.8 & 5.9 & 13.2 & 10.2 & 15.4 \\
\hline Transport and communications & 37.5 & 63.6 & 5.6 & 13.2 & 10.4 & 17.5 \\
\hline Finance and housing & 52.4 & 15.6 & 5.4 & 53.8 & 74.4 & 27.7 \\
\hline Public administration & 40.6 & 54.6 & 15.0 & 1.2 & 25.5 & 8.6 \\
\hline Other services & 52.5 & 16.4 & 54.9 & 13.6 & 10.0 & 12.8 \\
\hline GDP at factor costs & 43.2 & 18.7 & 13.9 & 51.0 & 53.8 & 13.0 \\
\hline Of which: non-oil GDP & 60.0 & 28.3 & 12.9 & 23.5 & 46.1 & 27.5 \\
\hline Import duties and subsidies & 14.9 & 8.8 & 31.9 & -0.3 & 6.6 & 2.3 \\
\hline GDP at market prices & 42.6 & 18.5 & 14.2 & 50.1 & 53.3 & 12.9 \\
\hline Of which: non-oil GDP & 55.3 & 26.8 & 14.2 & 21.7 & 43.7 & 26.3 \\
\hline
\end{tabular}

Sources: Equatoguinean authorities and IMF staff estimates. 
Table 2. Equatorial Guinea: GDP by Sector of Origin, 2001-06

(Percent of GDP, based on nominal magnitudes)

\begin{tabular}{|c|c|c|c|c|c|c|}
\hline & 2001 & 2002 & 2003 & 2004 & 2005 & $\begin{array}{r}2006 \\
\text { Est }\end{array}$ \\
\hline Primary sector & 84.5 & 82.6 & 82.0 & 84.9 & 84.9 & 82.6 \\
\hline Non-oil & 6.7 & 6.2 & 5.6 & 4.0 & 2.8 & 2.7 \\
\hline Agriculture & 3.9 & 3.8 & 3.5 & 2.6 & 1.9 & 1.8 \\
\hline Forestry & 2.6 & 2.3 & 2.0 & 1.3 & 0.9 & 0.8 \\
\hline Fishing & 0.1 & 0.1 & 0.1 & 0.1 & 0.1 & 0.1 \\
\hline Oil & 77.9 & 76.3 & 76.3 & 80.8 & 82.0 & 79.9 \\
\hline Secondary sector & 9.6 & 10.9 & 11.2 & 10.2 & 11.4 & 13.7 \\
\hline Manufacturing & 5.8 & 6.3 & 6.4 & 5.9 & 7.6 & 8.5 \\
\hline Oil derivatives & 5.6 & 6.1 & 6.2 & 5.7 & 7.5 & 8.4 \\
\hline Other manufacturing & 0.2 & 0.2 & 0.2 & 0.2 & 0.1 & 0.2 \\
\hline Electricity & 0.5 & 0.5 & 0.5 & 0.5 & 0.4 & 0.5 \\
\hline Construction & 3.3 & 4.2 & 4.3 & 3.9 & 3.4 & 4.6 \\
\hline Tertiary sector & 4.2 & 5.0 & 5.0 & 3.7 & 2.9 & 3.0 \\
\hline Trade and commerce & 1.5 & 1.7 & 1.6 & 1.2 & 0.9 & 0.9 \\
\hline Transport and communications & 0.2 & 0.3 & 0.3 & 0.2 & 0.1 & 0.1 \\
\hline Finance and housing & 0.3 & 0.3 & 0.3 & 0.3 & 0.3 & 0.4 \\
\hline Public administration & 1.5 & 2.0 & 2.0 & 1.3 & 1.1 & 1.1 \\
\hline Other services & 0.7 & 0.7 & 0.9 & 0.7 & 0.5 & 0.5 \\
\hline Import duties and subsidies & 1.7 & 1.6 & 1.8 & 1.2 & 0.8 & 0.8 \\
\hline Discrepancy & 0.0 & 0.0 & 0.0 & 0.0 & 0.0 & 0.0 \\
\hline GDP at market prices & 100.0 & 100.0 & 100.0 & 100.0 & 100.0 & 100.0 \\
\hline Of which: non-oil GDP & 22.1 & 23.7 & 23.7 & 19.2 & 18.0 & 20.1 \\
\hline
\end{tabular}

Sources: Equatoguinean authorities and IMF staff estimates. 
Table 3. Equatorial Guinea: GDP by Sector of Origin, 2001-06

\begin{tabular}{|c|c|c|c|c|c|c|}
\hline & 2001 & 2002 & 2003 & 2004 & 2005 & $\begin{array}{r}2006 \\
\text { Est. }\end{array}$ \\
\hline & \multicolumn{6}{|c|}{ (Billions of CFA francs in 2000 prices) } \\
\hline Primary sector & $1,253.6$ & $1,468.7$ & $1,653.1$ & $2,210.1$ & $2,281.9$ & $2,058.1$ \\
\hline Non-oil & 80.1 & 75.1 & 72.4 & 73.0 & 74.5 & 76.7 \\
\hline Agriculture & 44.6 & 45.9 & 43.3 & 45.0 & 47.3 & 50.1 \\
\hline Forestry & 34.0 & 27.2 & 26.9 & 25.8 & 24.9 & 24.2 \\
\hline Fishing & 1.5 & 2.0 & 2.2 & 2.2 & 2.4 & 2.4 \\
\hline Oil & $1,173.5$ & $1,393.6$ & $1,580.7$ & $2,137.1$ & $2,207.4$ & $1,981.4$ \\
\hline Secondary sector & 128.7 & 176.0 & 179.5 & 226.8 & 315.2 & 391.6 \\
\hline Manufacturing & 82.3 & 112.5 & 108.3 & 130.4 & 186.6 & 203.2 \\
\hline Oil derivatives & 80.0 & 109.9 & 105.2 & 126.6 & 182.2 & 198.2 \\
\hline Other manufacturing & 2.3 & 2.5 & 3.2 & 3.8 & 4.4 & 5.0 \\
\hline Electricity & 5.3 & 6.4 & 7.6 & 10.7 & 15.1 & 20.4 \\
\hline Construction & 41.1 & 57.1 & 63.5 & 85.7 & 113.6 & 168.1 \\
\hline Tertiary sector & 48.0 & 58.6 & 66.0 & 71.5 & 84.0 & 91.9 \\
\hline Trade and commerce & 17.4 & 23.0 & 22.7 & 24.6 & 25.9 & 28.6 \\
\hline Transport and communications & 2.1 & 2.9 & 2.9 & 3.2 & 3.4 & 3.8 \\
\hline Finance and housing & 3.5 & 4.1 & 4.2 & 6.2 & 10.7 & 13.1 \\
\hline Public administration & 16.4 & 18.9 & 21.5 & 21.7 & 27.4 & 28.5 \\
\hline Other services & 8.4 & 9.6 & 14.6 & 15.8 & 16.6 & 17.9 \\
\hline GDP at factor costs & $1,430.2$ & $1,703.3$ & $1,898.6$ & $2,508.4$ & $2,681.1$ & $2,541.7$ \\
\hline Import duties and subsidies & 15.6 & 15.1 & 18.8 & 17.3 & 15.1 & 14.6 \\
\hline GDP at market prices & $1,445.8$ & $1,718.4$ & $1,917.4$ & $2,525.7$ & $2,696.2$ & $2,556.3$ \\
\hline \multirow[t]{2}{*}{ Of which: non-oil GDP } & 272.4 & 324.8 & 336.7 & 388.6 & 488.8 & 574.9 \\
\hline & \multicolumn{6}{|c|}{ (Annual percentage change in constant prices) } \\
\hline Primary sector & 57.2 & 17.2 & 12.6 & 33.7 & 3.2 & -9.8 \\
\hline Non-oil & -6.7 & -6.2 & -3.6 & 0.8 & 2.1 & 3.0 \\
\hline Agriculture & -7.0 & 2.8 & -5.7 & 4.1 & 4.9 & 6.0 \\
\hline Forestry & -7.9 & -19.8 & -1.1 & -4.3 & -3.4 & -2.7 \\
\hline Fishing & 60.0 & 37.5 & 9.1 & 0.0 & 8.3 & 2.0 \\
\hline Oil & 64.9 & 18.8 & 13.4 & 35.2 & 3.3 & -10.2 \\
\hline Secondary sector & 224.9 & 36.7 & 2.0 & 26.4 & 39.0 & 24.2 \\
\hline Manufacturing & 562.3 & 36.6 & -3.7 & 20.3 & 43.1 & 8.9 \\
\hline Oil derivatives & 674.6 & 37.4 & -4.3 & 20.4 & 43.9 & 8.8 \\
\hline Other manufacturing & 10.0 & 9.1 & 25.0 & 20.0 & 16.7 & 13.0 \\
\hline Electricity & 30.0 & 20.5 & 19.1 & 41.1 & 40.5 & 35.0 \\
\hline Construction & 77.8 & 39.1 & 11.2 & 34.8 & 32.6 & 48.0 \\
\hline Tertiary sector & 29.1 & 22.1 & 12.7 & 8.3 & 17.4 & 9.5 \\
\hline Trade and commerce & 31.2 & 32.0 & -1.2 & 8.2 & 5.2 & 10.5 \\
\hline Transport and communications & 11.8 & 36.8 & 0.0 & 7.7 & 7.1 & 12.5 \\
\hline Finance and housing & 40.0 & 17.1 & 2.4 & 47.6 & 71.0 & 22.3 \\
\hline Public administration & 19.1 & 14.8 & 14.0 & 0.9 & 26.2 & 4.0 \\
\hline Other services & 50.0 & 14.3 & 52.1 & 8.2 & 5.1 & 8.0 \\
\hline GDP at factor costs & 63.6 & 19.1 & 11.5 & 32.1 & 6.9 & -5.2 \\
\hline Import duties and subsidies & -16.9 & -3.2 & 23.9 & -7.7 & -12.9 & -3.0 \\
\hline GDP at market prices & 61.9 & 18.8 & 11.6 & 31.7 & 6.7 & -5.2 \\
\hline Of which: non-oil GDP & 50.2 & 19.2 & 3.7 & 15.4 & 25.8 & 17.6 \\
\hline
\end{tabular}

Sources: Equatoguinean authorities and IMF staff estimates. 
Table 4. Equatorial Guinea: GDP by Use of Resources, 2001-06

\begin{tabular}{|c|c|c|c|c|c|c|}
\hline & 2001 & 2002 & 2003 & 2004 & 2005 & $\begin{array}{r}2006 \\
\text { Est. }\end{array}$ \\
\hline & \multicolumn{6}{|c|}{ (Billions of CFA francs) } \\
\hline Domestic demand & $1,155.7$ & 802.3 & $1,373.6$ & $1,592.5$ & $2,018.4$ & $2,493.4$ \\
\hline Resource balance & 117.9 & 707.4 & 350.5 & 995.5 & $1,948.4$ & $1,984.2$ \\
\hline Exports of goods and nonfactor services & $1,289.9$ & $1,490.6$ & $1,661.8$ & $2,518.0$ & $3,838.5$ & $4,229.9$ \\
\hline Imports of goods and nonfactor services & $-1,171.9$ & -783.2 & $-1,311.4$ & $-1,522.5$ & $-1,890.1$ & $-2,245.7$ \\
\hline Gross domestic product & $1,273.6$ & $1,509.6$ & $1,724.1$ & $2,588.0$ & $3,966.8$ & $4,477.6$ \\
\hline Net factor income from abroad & -636.5 & -698.5 & -909.2 & $-1,537.9$ & $-2,133.1$ & $-1,738.7$ \\
\hline Public & -4.9 & -2.6 & -2.3 & -3.5 & -7.1 & -3.4 \\
\hline Private & -631.6 & -695.9 & -906.9 & $-1,534.4$ & $-2,126.0$ & $-1,735.3$ \\
\hline Gross national product & 637.1 & 811.1 & 814.9 & $1,050.2$ & $1,833.7$ & $2,739.0$ \\
\hline Unrequited transfers & -1.5 & -7.7 & -15.2 & -24.3 & -35.2 & -48.3 \\
\hline Private & 10.6 & 10.6 & 10.6 & 10.6 & 10.6 & 10.6 \\
\hline Public & -12.1 & -18.3 & -25.8 & -34.8 & -45.7 & -58.8 \\
\hline Gross disposable income & 635.6 & 803.4 & 799.6 & $1,025.9$ & $1,798.5$ & $2,690.7$ \\
\hline Consumption & 244.9 & 330.2 & 367.7 & 440.7 & 521.4 & 675.7 \\
\hline Public & 41.4 & 76.9 & 65.0 & 81.3 & 118.6 & 128.1 \\
\hline Private & 203.5 & 253.3 & 302.7 & 359.4 & 402.8 & 547.5 \\
\hline National savings & 390.7 & 473.2 & 432.0 & 585.2 & $1,277.1$ & $2,015.0$ \\
\hline Gross capital formation & 910.8 & 472.1 & $1,005.9$ & $1,151.8$ & $1,497.0$ & $1,817.7$ \\
\hline Public & 94.0 & 126.6 & 169.8 & 363.0 & 400.7 & 675.9 \\
\hline Private & 816.6 & 345.4 & 835.9 & 788.6 & $1,096.1$ & $1,141.7$ \\
\hline Oil & 779.1 & 281.8 & 759.4 & 681.3 & 973.0 & 712.8 \\
\hline Non-oil & 37.5 & 63.6 & 76.5 & 107.3 & 123.0 & 428.9 \\
\hline Change in inventories & 0.2 & 0.1 & 0.2 & 0.2 & 0.2 & 0.2 \\
\hline \multirow[t]{2}{*}{ External current account } & -520.1 & 1.1 & -574.0 & -566.7 & -219.9 & 197.3 \\
\hline & \multicolumn{6}{|c|}{ (Percent of GDP) } \\
\hline Domestic demand & 90.7 & 53.1 & 79.7 & 61.5 & 50.9 & 55.7 \\
\hline Resource balance & 9.3 & 46.9 & 20.3 & 38.5 & 49.1 & 44.3 \\
\hline Net factor income from abroad & -50.0 & -46.3 & -52.7 & -59.4 & -53.8 & -38.8 \\
\hline Unrequited transfers & -0.1 & -0.5 & -0.9 & -0.9 & -0.9 & -1.1 \\
\hline Gross disposable income & 49.9 & 53.2 & 46.4 & 39.6 & 45.3 & 60.1 \\
\hline Consumption & 19.2 & 21.9 & 21.3 & 17.0 & 13.1 & 15.1 \\
\hline Public & 3.3 & 5.1 & 3.8 & 3.1 & 3.0 & 2.9 \\
\hline Private & 16.0 & 16.8 & 17.6 & 13.9 & 10.2 & 12.2 \\
\hline National savings & 30.7 & 31.3 & 25.1 & 22.6 & 32.2 & 45.0 \\
\hline Gross fixed capital formation & 71.5 & 31.3 & 58.3 & 44.5 & 37.7 & 40.6 \\
\hline Public & 7.4 & 8.4 & 9.8 & 14.0 & 10.1 & 15.1 \\
\hline Private & 64.1 & 22.9 & 48.5 & 30.5 & 27.6 & 25.5 \\
\hline External current account & -40.8 & 0.1 & -33.3 & -21.9 & -5.5 & 4.4 \\
\hline
\end{tabular}

Sources: Equaguinean authorities and IMF staff estimates. 
Table 5. Equatorial Guinea: Consumer Price Index, 2001-06

\begin{tabular}{lrrrrrrr}
\hline & $\begin{array}{r}\text { Weight } \\
\text { (percent) }\end{array}$ & $\begin{array}{r}2001 \\
\text { Dec. }\end{array}$ & $\begin{array}{r}2002 \\
\text { Dec. }\end{array}$ & $\begin{array}{r}2003 \\
\text { Dec. }\end{array}$ & $\begin{array}{r}2004 \\
\text { Dec. }\end{array}$ & $\begin{array}{r}2005 \\
\text { Dec }\end{array}$ & $\begin{array}{r}2006 \\
\text { Dec }\end{array}$ \\
\hline & & & & (Index, $2000=100)$ \\
Food, beverages and tobacco & 60 & 116.1 & 125.3 & 130.1 & 141.6 & 146.6 & 153.1 \\
Clothing & 16 & 105.9 & 108.6 & 125.2 & 128.8 & 133.7 & 135.3 \\
Housing, water, electricity & 5 & 104.3 & 99.4 & 106.0 & 108.2 & 109.3 & 111.3 \\
Furniture and other equipments & 9 & 105.4 & 112.3 & 119.7 & 123.7 & 129.5 & 132.9 \\
Health & 1 & 100.4 & 104.0 & 104.0 & 105.2 & 110.8 & 111.7 \\
Transport & 3 & 124.0 & 120.9 & 119.8 & 139.6 & 149.3 & 144.8 \\
Leisure & 1 & 102.0 & 105.0 & 106.7 & 106.9 & 107.0 & 102.7 \\
Hotel, restaurants & 3 & 102.3 & 117.2 & 134.5 & 141.1 & 150.2 & 155.4 \\
Other goods and services & 1 & 103.5 & 114.0 & 114.4 & 113.4 & 114.8 & 115.9 \\
General index & 100 & 112.3 & 119.2 & 126.2 & 134.9 & 139.2 & 144.5 \\
\hline
\end{tabular}

Source: Equatoguinean authorities.

Table 6. Equatorial Guinea: Consumer Price Inflation, 2001-06

\begin{tabular}{|c|c|c|c|c|c|c|}
\hline & 2001 & 2002 & 2003 & 2004 & 2005 & 2006 \\
\hline & \multicolumn{6}{|c|}{ (12-month percent changes) } \\
\hline January & 4.6 & 7.6 & 4.8 & 3.1 & 7.4 & 3.5 \\
\hline February & 4.6 & 7.7 & 9.7 & 1.6 & 10.4 & 1.7 \\
\hline March & 5.7 & 7.8 & 9.5 & 3.7 & 7.3 & 4.1 \\
\hline April & 6.8 & 9.1 & 9.3 & 3.7 & 7.1 & 4.2 \\
\hline May & 9.7 & 6.7 & 8.5 & 4.6 & 5.7 & 4.5 \\
\hline June & 8.9 & 8.7 & 9.5 & 4.3 & 5.2 & 5.2 \\
\hline July & 8.6 & 7.7 & 7.5 & 4.1 & 4.5 & 5.7 \\
\hline August & 8.2 & 7.1 & 6.7 & 5.1 & 4.4 & 5.2 \\
\hline September & 11.8 & 6.2 & 5.5 & 5.7 & 4.0 & 5.4 \\
\hline October & 12.3 & 5.8 & 6.1 & 4.6 & 5.0 & 5.5 \\
\hline November & 12.4 & 6.2 & 5.5 & 5.1 & 4.0 & 4.7 \\
\hline \multirow[t]{2}{*}{ December } & 12.3 & 4.8 & 5.9 & 5.1 & 3.2 & 3.8 \\
\hline & \multicolumn{6}{|c|}{ (Annual percentage change) } \\
\hline Average & 8.8 & 7.6 & 7.4 & 4.2 & 5.7 & 4.5 \\
\hline
\end{tabular}

Source: Equatoguinean authorities and IMF staff estimates. 
Table 7. Equatorial Guinea: Summary of Central Government Financial Operations, 2001-06

(Billions of CFA francs)

\begin{tabular}{|c|c|c|c|c|c|c|}
\hline & 2001 & 2002 & 2003 & 2004 & 2005 & 2006 \\
\hline Total revenue and grants & 348.0 & 414.5 & 471.2 & 824.3 & $1,504.1$ & $2,047.0$ \\
\hline Revenue & 348.0 & 414.5 & 471.2 & 824.3 & $1,504.1$ & $2,047.0$ \\
\hline Tax revenue & 102.0 & 188.9 & 175.5 & 226.6 & 371.0 & 510.0 \\
\hline Taxes on income, profits, and capital gains & 79.2 & 164.3 & 142.9 & 190.2 & 337.9 & 466.9 \\
\hline Personal income tax & 22.5 & 24.2 & 17.5 & 26.2 & 33.3 & 34.4 \\
\hline Corporate income tax & 56.4 & 125.4 & 125.2 & 163.7 & 304.4 & 432.3 \\
\hline Other income taxes & 0.2 & 14.7 & 0.2 & 0.3 & 0.1 & 0.2 \\
\hline Domestic taxes on goods and services & 12.3 & 14.2 & 19.7 & 22.6 & 22.1 & 28.8 \\
\hline Taxes on international trade and transactions & 9.3 & 9.3 & 11.3 & 8.3 & 8.1 & 10.2 \\
\hline Other taxes & 1.2 & 1.1 & 1.6 & 5.5 & 2.9 & 4.0 \\
\hline Nontax revenue & 246.0 & 225.6 & 295.6 & 597.7 & $1,133.0$ & $1,537.1$ \\
\hline Hydrocarbons sector & 233.1 & 212.9 & 279.6 & 580.9 & $1,089.3$ & $1,475.9$ \\
\hline Non hydrocarbons sector & 12.9 & 12.7 & 16.0 & 16.7 & 43.7 & 61.1 \\
\hline Interest on saving funds & 0.0 & 0.0 & 0.0 & 0.0 & 12.6 & 17.2 \\
\hline Grants & 0.0 & 0.0 & 0.0 & 0.0 & 0.0 & 0.0 \\
\hline Total expenditure and net lending & 165.9 & 229.4 & 270.7 & 501.6 & 736.5 & 997.2 \\
\hline Current expenditure & 63.8 & 100.6 & 99.1 & 121.1 & 163.3 & 199.4 \\
\hline Wages and salaries & 17.4 & 26.0 & 27.5 & 30.9 & 36.6 & 40.6 \\
\hline Goods and services & 24.0 & 50.9 & 49.7 & 50.5 & 82.0 & 87.5 \\
\hline Interest payments & 7.1 & 3.9 & 1.8 & 1.7 & 1.7 & 1.2 \\
\hline Transfers and subsidies & 15.3 & 19.8 & 20.1 & 38.0 & 43.0 & 70.2 \\
\hline Capital expenditure & 94.0 & 126.6 & 169.7 & 378.8 & 560.6 & 795.2 \\
\hline Net lending & 0.0 & 0.0 & 0.0 & 0.0 & 0.0 & 0.0 \\
\hline Domestic arrears payments & 8.1 & 2.1 & 1.9 & 1.7 & 12.6 & 2.6 \\
\hline Reimbursement to depositors $^{1}$ & 8.1 & 2.1 & 1.9 & 1.7 & 12.6 & 2.6 \\
\hline Overall balance & 182.1 & 185.1 & 200.5 & 322.7 & 767.5 & $1,049.8$ \\
\hline Total financing & -182.1 & -185.1 & -200.5 & -322.7 & -767.5 & $-1,049.8$ \\
\hline Foreign borrowing (net) & -84.7 & -180.3 & -96.9 & -55.8 & -74.8 & -721.0 \\
\hline Budget support loans & 0.0 & 0.0 & 0.0 & 0.0 & 0.0 & 0.0 \\
\hline Amortization (-) & -5.7 & -5.8 & -3.5 & -4.2 & -19.1 & -49.8 \\
\hline Exceptional financing & -79.0 & -174.5 & -93.4 & -51.6 & -55.7 & -671.2 \\
\hline Payment of external arrears (-) & 4.0 & 0.0 & 0.0 & 0.0 & 0.0 & 0.0 \\
\hline Debt rescheduling & 1.3 & 0.0 & 0.0 & 0.0 & 0.0 & 0.0 \\
\hline Treasury deposits abroad (-=increase) & -84.3 & -174.5 & -93.4 & -16.6 & -60.7 & -578.5 \\
\hline Gepetrol/Sonagas deposits abroad (-=increase) & 0.0 & 0.0 & 0.0 & -35.0 & 5.0 & -92.7 \\
\hline Domestic borrowing (net) & -35.8 & -22.8 & 16.5 & -284.7 & -695.4 & -326.6 \\
\hline Errors and omissions/financing gap & -61.7 & 17.9 & -120.0 & 17.8 & 2.7 & -2.2 \\
\hline \multicolumn{7}{|l|}{ Memorandum items: } \\
\hline Overall balance, excluding grants & 182.1 & 185.1 & 200.5 & 322.7 & 767.5 & $1,049.8$ \\
\hline in percent of GDP & 14.3 & 12.3 & 11.6 & 12.5 & 19.3 & 23.4 \\
\hline Non oil primary balance, excluding grants ${ }^{2}$ & -96.9 & -149.2 & -181.1 & -367.0 & -458.2 & -580.0 \\
\hline in percent of non-oil GDP & -34.4 & -41.8 & -44.4 & -73.9 & -64.3 & -64.4 \\
\hline Nominal GDP & $1,273.6$ & $1,509.6$ & $1,724.1$ & $2,588.0$ & $3,966.8$ & $4,477.6$ \\
\hline Nominal non-oil GDP & 281.7 & 357.1 & 407.8 & 496.4 & 713.0 & 900.7 \\
\hline
\end{tabular}

Sources: data provided by the Equatoguinean authorities, and IMF estimates and projections.

${ }^{1}$ Related to the crash of the banking system during the early eighties.

${ }^{2}$ Equal to the overall balance excluding grants minus hydrocarbons sector corporate income tax and non tax revenue plus hydrocarbons revenue generated in the secondary LNG, LPG and methanol production and purchase of share in hydrocarbons projects, minus interest on saving funds plus interest expenditure. 
Table 8. Equatorial Guinea: Detailed Central Government Revenue, 2001-06

(Billions of CFA francs)

\begin{tabular}{|c|c|c|c|c|c|c|}
\hline & 2001 & 2002 & 2003 & 2004 & 2005 & 2006 \\
\hline Total revenue and grants & 348.0 & 414.5 & 471.2 & 824.3 & $1,504.1$ & $2,047.0$ \\
\hline Revenue & 348.0 & 414.5 & 471.2 & 824.3 & $1,504.1$ & $2,047.0$ \\
\hline Tax revenue & 102.0 & 188.9 & 175.5 & 226.6 & 371.0 & 510.0 \\
\hline Taxes on income, profits, and capital gains & 79.2 & 164.3 & 142.9 & 190.2 & 337.9 & 466.9 \\
\hline Personal income tax & 22.5 & 24.2 & 17.5 & 26.2 & 33.3 & 34.4 \\
\hline Hydrocarbons sector & 17.7 & 24.2 & 11.9 & 19.8 & 25.9 & 23.6 \\
\hline Non hydrocarbons sector & 4.9 & 0.0 & 5.7 & 6.5 & 7.4 & 10.7 \\
\hline Corporate income tax & 56.4 & 125.4 & 125.2 & 163.7 & 304.4 & 432.3 \\
\hline Hydrocarbons sector & 53.0 & 125.4 & 118.2 & 150.4 & 286.2 & 406.5 \\
\hline Contractors & 47.9 & 112.4 & 110.6 & 118.2 & 67.2 & 331.7 \\
\hline Sub-contractors & 5.2 & 13.0 & 7.6 & 32.2 & 219.0 & 74.7 \\
\hline Non hydrocarbons sector & 3.4 & 0.0 & 7.0 & 13.3 & 18.3 & 25.9 \\
\hline Other income taxes & 0.2 & 14.7 & 0.2 & 0.3 & 0.1 & 0.2 \\
\hline Domestic taxes on goods and services & 12.3 & 14.2 & 19.7 & 22.6 & 22.1 & 28.8 \\
\hline Value added tax ${ }^{1}$ & 7.9 & 0.0 & 14.6 & 15.1 & 15.0 & 21.3 \\
\hline Petroleum taxes & 4.4 & 0.0 & 5.1 & 7.5 & 7.1 & 7.5 \\
\hline Other domestic taxes on goods and services & 0.0 & 14.2 & 0.0 & 0.0 & 0.0 & 0.0 \\
\hline Taxes on international trade and transactions & 9.3 & 9.3 & 11.3 & 8.3 & 8.1 & 10.2 \\
\hline Import tariffs & 3.6 & 4.9 & 4.9 & 4.4 & 4.0 & 5.3 \\
\hline Export taxes & 5.7 & 4.4 & 6.4 & 3.9 & 4.1 & 4.9 \\
\hline Other taxes & 1.2 & 1.1 & 1.6 & 5.5 & 2.9 & 4.0 \\
\hline Nontax revenue & 246.0 & 225.6 & 295.6 & 597.7 & $1,133.0$ & $1,537.1$ \\
\hline Hydrocarbons sector & 233.1 & 212.9 & 279.6 & 580.9 & $1,089.3$ & $1,475.9$ \\
\hline Royalties & 197.2 & 172.2 & 240.1 & 381.6 & 462.6 & 970.0 \\
\hline Profit sharing & 34.7 & 40.7 & 38.6 & 195.3 & 573.4 & 500.2 \\
\hline Primary oil and gas production & 34.7 & 40.7 & 24.1 & 171.1 & 527.4 & 359.1 \\
\hline Secondary LNG, LPG, methanol production & 0.0 & 0.0 & 14.5 & 24.1 & 46.0 & 141.1 \\
\hline Bonuses and rents & 1.2 & 0.0 & 0.9 & 4.1 & 53.2 & 5.8 \\
\hline Non hydrocarbons sector & 12.9 & 12.7 & 16.0 & 16.7 & 43.7 & 61.1 \\
\hline Nontax revenue excluding interest on saving funds & 12.9 & 12.7 & 16.0 & 16.7 & 31.2 & 43.9 \\
\hline Interest on saving funds & 0.0 & 0.0 & 0.0 & 0.0 & 12.6 & 17.2 \\
\hline Grants & 0.0 & 0.0 & 0.0 & 0.0 & 0.0 & 0.0 \\
\hline
\end{tabular}

Sources: Data provided by the Equatoguinean authorities, and IMF staff estimates and projections.

${ }^{1}$ The VAT was legislated in early 2005; previously this was a sales tax. Currently, 40 percent of firms in the non hydrocarbons sector continue to pay on the basis of a sales tax. 
Table 9. Equatorial Guinea: Detailed Central Government Expenditure and Financing, 2001-06 (Billions of CFA francs)

\begin{tabular}{|c|c|c|c|c|c|c|}
\hline & 2001 & 2002 & 2003 & 2004 & 2005 & 2006 \\
\hline Total expenditure and net lending & 165.9 & 229.4 & 270.7 & 501.6 & 736.5 & 997.2 \\
\hline Current expenditure & 63.8 & 100.6 & 99.1 & 121.1 & 163.3 & 199.4 \\
\hline Wages and salaries & 17.4 & 26.0 & 27.5 & 30.9 & 36.6 & 40.6 \\
\hline Goods and services & 24.0 & 50.9 & 49.7 & 50.5 & 82.0 & 87.5 \\
\hline Interest payments & 7.1 & 3.9 & 1.8 & 1.7 & 1.7 & 1.2 \\
\hline Domestic & 1.2 & 1.4 & 0.2 & 0.4 & 0.5 & 0.3 \\
\hline Foreign & 5.8 & 2.6 & 1.6 & 1.3 & 1.2 & 0.8 \\
\hline Transfers and subsidies & 15.3 & 19.8 & 20.1 & 38.0 & 43.0 & 70.2 \\
\hline Transfers & 1.5 & 0.0 & 3.0 & 5.5 & 0.7 & 5.6 \\
\hline Subsidies & 13.7 & 19.8 & 17.1 & 32.6 & 42.3 & 64.5 \\
\hline Other current expenditure & 0.0 & 0.0 & 0.0 & 0.0 & 0.0 & 0.0 \\
\hline Capital expenditure & 94.0 & 126.6 & 169.7 & 378.8 & 560.6 & 795.2 \\
\hline Foreign financed & 0.0 & 0.0 & 0.0 & 0.0 & 0.0 & 0.0 \\
\hline Domestically financed & 94.0 & 126.6 & 169.7 & 378.8 & 560.6 & 795.2 \\
\hline Purchase of share in hydrocarbons projects & 0.0 & 0.0 & 0.0 & 15.8 & 114.6 & 127.7 \\
\hline Net lending & 0.0 & 0.0 & 0.0 & 0.0 & 0.0 & 0.0 \\
\hline Domestic arrears payments & 8.1 & 2.1 & 1.9 & 1.7 & 12.6 & 2.6 \\
\hline Reimbursement to depositors ${ }^{1}$ & 8.1 & 2.1 & 1.9 & 1.7 & 12.6 & 2.6 \\
\hline Overall balance & 182.1 & 185.1 & 200.5 & 322.7 & 767.5 & $1,049.8$ \\
\hline Total financing & -182.1 & -185.1 & -200.5 & -322.7 & -767.5 & $-1,049.8$ \\
\hline Foreign borrowing (net) & -84.7 & -180.3 & -96.9 & -55.8 & -74.8 & -721.0 \\
\hline Budget support loans & 0.0 & 0.0 & 0.0 & 0.0 & 0.0 & 0.0 \\
\hline Amortization $(-)$ & -5.7 & -5.8 & -3.5 & -4.2 & -19.1 & -49.8 \\
\hline Exceptional financing & -79.0 & -174.5 & -93.4 & -51.6 & -55.7 & -671.2 \\
\hline Payment of external arrears (-) & 4.0 & 0.0 & 0.0 & 0.0 & 0.0 & 0.0 \\
\hline Debt rescheduling & 1.3 & 0.0 & 0.0 & 0.0 & 0.0 & 0.0 \\
\hline Treasury deposits abroad (-=increase) & -84.3 & -174.5 & -93.4 & -16.6 & -60.7 & -578.5 \\
\hline Gepetrol/Sonagas deposits abroad (-=increase) & 0.0 & 0.0 & 0.0 & -35.0 & 5.0 & -92.7 \\
\hline Domestic borrowing (net) & -35.8 & -22.8 & 16.5 & -284.7 & -695.4 & -326.6 \\
\hline Errors and omissions/financing gap & -61.7 & 17.9 & -120.0 & 17.8 & 2.7 & -2.2 \\
\hline
\end{tabular}

Sources: data provided by the Equatoguinean authorities, and IMF staff estimates and projections.

${ }^{1}$ Related to the crash of the banking system during the early eighties. 
Table 10. Equatorial Guinea: Fiscal Indicators, 2001-06

\begin{tabular}{|c|c|c|c|c|c|c|}
\hline & 2001 & 2002 & 2003 & 2004 & 2005 & 2006 \\
\hline & \multicolumn{6}{|c|}{ (Billions of CFA francs) } \\
\hline Total revenue & 348.0 & 414.5 & 471.2 & 824.3 & $1,504.1$ & $2,047.0$ \\
\hline Oil revenue & 286.1 & 338.3 & 383.3 & 707.2 & $1,329.5$ & $1,741.3$ \\
\hline Non-oil revenue (including oil derivatives) & 61.9 & 76.2 & 87.8 & 117.1 & 174.6 & 305.7 \\
\hline Total expenditure and net lending & 165.9 & 229.4 & 270.7 & 501.6 & 736.5 & 997.2 \\
\hline Current expenditure & 63.8 & 100.6 & 99.1 & 121.1 & 163.3 & 199.4 \\
\hline Wages and salaries & 17.4 & 26.0 & 27.5 & 30.9 & 36.6 & 40.6 \\
\hline Goods and services & 24.0 & 50.9 & 49.7 & 50.5 & 82.0 & 87.5 \\
\hline Subsidies and transfers & 15.3 & 19.8 & 20.1 & 38.0 & 43.0 & 70.2 \\
\hline Scheduled interest & 7.1 & 3.9 & 1.8 & 1.7 & 1.7 & 1.2 \\
\hline Capital expenditure & 94.0 & 126.6 & 169.7 & 378.8 & 560.6 & 795.2 \\
\hline Overall balance, excluding grants & 182.1 & 185.1 & 200.5 & 322.7 & 767.5 & $1,049.8$ \\
\hline \multirow[t]{2}{*}{ Non-oil primary balance, excluding grants } & -96.9 & -149.2 & -181.1 & -367.0 & -458.2 & -580.0 \\
\hline & \multicolumn{6}{|c|}{ (Annual percentage change) } \\
\hline Total revenue & 111.7 & 19.1 & 13.7 & 75.0 & 82.5 & 36.1 \\
\hline Oil revenue & 111.4 & 18.2 & 13.3 & 84.5 & 88.0 & 31.0 \\
\hline Non-oil revenue (including oil derivatives) & 112.9 & 23.1 & 15.2 & 33.3 & 49.1 & 75.1 \\
\hline Total expenditure and net lending & 39.4 & 38.2 & 18.0 & 85.3 & 46.8 & 35.4 \\
\hline Current expenditure & 6.9 & 57.7 & -1.5 & 22.3 & 34.8 & 22.1 \\
\hline Wages and salaries & 34.8 & 49.3 & 5.7 & 12.2 & 18.6 & 10.9 \\
\hline Goods and services & -22.4 & 112.0 & -2.3 & 1.6 & 62.4 & 6.8 \\
\hline Subsidies and transfers & 25.6 & 29.3 & 1.5 & 89.5 & 13.1 & 63.1 \\
\hline Scheduled interest & 91.8 & -44.6 & -54.4 & -3.7 & -1.9 & -31.0 \\
\hline Capital expenditure & 70.8 & 34.7 & 34.0 & 123.2 & 48.0 & 41.8 \\
\hline Overall balance, excluding grants & 301.2 & 1.7 & 8.3 & 61.0 & 137.9 & 36.8 \\
\hline \multirow[t]{2}{*}{ Non-oil primary balance, excluding grants } & 12.4 & 53.9 & 21.4 & 102.7 & 24.8 & 26.6 \\
\hline & \multicolumn{6}{|c|}{ (Percent of total revenue) } \\
\hline Total revenue & 100.0 & 100.0 & 100.0 & 100.0 & 100.0 & 100.0 \\
\hline Oil revenue & 82.2 & 81.6 & 81.4 & 85.8 & 88.4 & 85.1 \\
\hline \multirow[t]{2}{*}{ Non-oil revenue (including oil derivatives) } & 17.8 & 18.4 & 18.6 & 14.2 & 11.6 & 14.9 \\
\hline & \multicolumn{6}{|c|}{ (Percent of total expenditure) } \\
\hline Total expenditure and net lending & 100.0 & 100.0 & 100.0 & 100.0 & 100.0 & 100.0 \\
\hline Current expenditure & 38.5 & 43.9 & 36.6 & 24.1 & 22.2 & 20.0 \\
\hline Wages and salaries & 10.5 & 11.4 & 10.2 & 6.2 & 5.0 & 4.1 \\
\hline Goods and services & 14.5 & 22.2 & 18.3 & 10.1 & 11.1 & 8.8 \\
\hline Subsidies and transfers & 9.2 & 8.6 & 7.4 & 7.6 & 5.8 & 7.0 \\
\hline Scheduled interest & 4.3 & 1.7 & 0.7 & 0.3 & 0.2 & 0.1 \\
\hline \multirow[t]{2}{*}{ Capital expenditure } & 56.6 & 55.2 & 62.7 & 75.5 & 76.1 & 79.7 \\
\hline & \multicolumn{6}{|c|}{ (Percent of GDP) } \\
\hline Total revenue & 27.3 & 27.5 & 27.3 & 31.9 & 37.9 & 45.7 \\
\hline Oil revenue & 22.5 & 22.4 & 22.2 & 27.3 & 33.5 & 38.9 \\
\hline Non-oil revenue (including oil derivatives) & 4.9 & 5.0 & 5.1 & 4.5 & 4.4 & 6.8 \\
\hline Total expenditure and net lending & 13.0 & 15.2 & 15.7 & 19.4 & 18.6 & 22.3 \\
\hline Current expenditure & 5.0 & 6.7 & 5.7 & 4.7 & 4.1 & 4.5 \\
\hline Wages and salaries & 1.4 & 1.7 & 1.6 & 1.2 & 0.9 & 0.9 \\
\hline Goods and services & 1.9 & 3.4 & 2.9 & 1.9 & 2.1 & 2.0 \\
\hline Subsidies and transfers & 1.2 & 1.3 & 1.2 & 1.5 & 1.1 & 1.6 \\
\hline Scheduled interest & 0.6 & 0.3 & 0.1 & 0.1 & 0.0 & 0.0 \\
\hline Capital expenditure & 7.4 & 8.4 & 9.8 & 14.6 & 14.1 & 17.8 \\
\hline \multirow[t]{2}{*}{ Overall balance, excluding grants } & 14.3 & 12.3 & 11.6 & 12.5 & 19.3 & 23.4 \\
\hline & \multicolumn{6}{|c|}{ (Percent of non-oil GDP) } \\
\hline Non-oil revenue (including oil derivatives) & 22.0 & 21.3 & 21.5 & 23.6 & 24.5 & 33.9 \\
\hline Non-oil primary balance, excluding grants & -34.4 & -41.8 & -44.4 & -73.9 & -64.3 & -64.4 \\
\hline
\end{tabular}

Sources: Equatoguinean authorities and IMF staff estimates. 
Table 11. Equatorial Guinea: Public Investment Program, 2004-06 (Execution) and 2007-08 (Budgeted)

\begin{tabular}{|c|c|c|c|c|c|}
\hline & 2004 & 2005 & 2006 & 2007 & 2008 \\
\hline & & & & \multicolumn{2}{|c|}{ Budgeted } \\
\hline Expenditure by sector & \multicolumn{5}{|c|}{ (Millions of CFA francs) } \\
\hline Administrative development $^{1}$ & 65,346 & 117,237 & 137,396 & 263,305 & 212,468 \\
\hline Infrastructure development $^{2}$ & 225,082 & 257,228 & 378,138 & 647,437 & 564,718 \\
\hline Productive development $^{3}$ & 7,261 & 12,916 & 184,253 & 11,428 & 6,892 \\
\hline Social development $^{4}$ & 65,346 & 58,622 & 95,375 & 57,267 & 23,658 \\
\hline Total & 363,035 & 446,003 & 795,162 & 979,436 & 807,736 \\
\hline Expenditure by sector & \multicolumn{5}{|c|}{ (Millions of U.S. dollars) } \\
\hline Administrative development ${ }^{1}$ & 123.7 & 222.5 & 263.0 & 522.1 & 423.2 \\
\hline Infrastructure development ${ }^{2}$ & 426.1 & 488.2 & 723.8 & 1283.9 & 1124.7 \\
\hline Productive development $^{3}$ & 13.7 & 24.5 & 352.7 & 22.7 & 13.7 \\
\hline Social development ${ }^{4}$ & 123.7 & 111.3 & 182.6 & 113.6 & 47.1 \\
\hline Total & 687.2 & 846.5 & 1522.0 & 1942.3 & 1608.7 \\
\hline Expenditure by sector & \multicolumn{5}{|c|}{ (Percent of total capital expenditure) } \\
\hline Administrative development ${ }^{1}$ & 18.0 & 26.3 & 17.3 & 26.9 & 26.3 \\
\hline Infrastructure development ${ }^{2}$ & 62.0 & 57.7 & 47.6 & 66.1 & 69.9 \\
\hline Productive development ${ }^{3}$ & 2.0 & 2.9 & 23.2 & 1.2 & 0.9 \\
\hline Social development ${ }^{4}$ & 18.0 & 13.1 & 12.0 & 5.8 & 2.9 \\
\hline Total & 100.0 & 100.0 & 100.0 & 100.0 & 100.0 \\
\hline
\end{tabular}

Source: Equatorial Guinean authorities.

${ }^{1}$ Includes general public administration, defense and national security, judiciary, and local governments.

${ }^{2}$ Includes transport and communications, housing and urban development, water and sewerage, and energy.

${ }^{3}$ Includes agriculture and livestock, rural development, fishing, environment, tourism, and industry and commerce.

${ }^{4}$ Includes education, science, sports, health, gender, information, culture, and social network. 
Table 12. Equatorial Guinea: Monetary Survey, 2001-06

\begin{tabular}{|c|c|c|c|c|c|c|}
\hline & $\begin{array}{l}2001 \\
\text { Dec. }\end{array}$ & $\begin{array}{l}2002 \\
\text { Dec. }\end{array}$ & $\begin{array}{l}2003 \\
\text { Dec. }\end{array}$ & $\begin{array}{l}2004 \\
\text { Dec. }\end{array}$ & $\begin{array}{l}2005 \\
\text { Dec. }\end{array}$ & $\begin{array}{l}2006 \\
\text { Dec. }\end{array}$ \\
\hline & \multicolumn{6}{|c|}{ (In billions of CFA francs) } \\
\hline \multicolumn{7}{|l|}{ Monetary survey } \\
\hline $\begin{array}{l}\text { Net foreign assets } \\
\text { (in millions of U.S. dollars) }\end{array}$ & $\begin{array}{l}60.6 \\
82.4\end{array}$ & $\begin{array}{l}104.6 \\
162.3\end{array}$ & $\begin{array}{l}153.6 \\
287.8\end{array}$ & $\begin{array}{r}499.6 \\
1,021.0\end{array}$ & $\begin{array}{l}1,241.0 \\
2,242.9\end{array}$ & $\begin{array}{l}1,573.9 \\
3,160.1\end{array}$ \\
\hline $\begin{array}{l}\text { Net domestic assets } \\
\text { Domestic credit } \\
\text { Claims on government (net) } \\
\text { Claims on nongovernment } \\
\text { Other items (net) }\end{array}$ & $\begin{array}{r}4.1 \\
8.8 \\
-28.0 \\
36.9 \\
-4.7\end{array}$ & $\begin{array}{r}-5.5 \\
3.4 \\
-50.8 \\
54.2 \\
-8.9\end{array}$ & $\begin{array}{r}1.6 \\
17.3 \\
-34.3 \\
51.6 \\
-15.6\end{array}$ & $\begin{array}{r}-292.4 \\
-269.2 \\
-332.0 \\
62.8 \\
-23.2\end{array}$ & $\begin{array}{r}-961.9 \\
-933.6 \\
-1,027.4 \\
93.8 \\
-28.3\end{array}$ & $\begin{array}{r}-1,255.4 \\
-1,224.6 \\
-1,354.0 \\
129.5 \\
-30.8\end{array}$ \\
\hline $\begin{array}{l}\text { Broad money (M2) } \\
\text { Currency } \\
\text { Deposits }\end{array}$ & $\begin{array}{l}64.7 \\
17.6 \\
47.1\end{array}$ & $\begin{array}{l}99.1 \\
26.0 \\
73.1\end{array}$ & $\begin{array}{r}155.2 \\
35.1 \\
120.1\end{array}$ & $\begin{array}{r}207.2 \\
45.7 \\
161.4\end{array}$ & $\begin{array}{r}279.1 \\
57.7 \\
221.4\end{array}$ & $\begin{array}{r}318.5 \\
68.2 \\
250.3\end{array}$ \\
\hline Deposits & \multicolumn{6}{|c|}{ (Annual percentage change, unless otherwise specified) } \\
\hline \multicolumn{7}{|l|}{ Memorandum items: } \\
\hline CPI inflation (average annual) & 8.8 & 7.6 & 7.3 & 4.2 & 5.7 & 4.5 \\
\hline Broad money (M2) & 35.1 & 53.1 & 56.7 & 33.5 & 34.7 & 14.1 \\
\hline Reserve money (RM) & 85.6 & 4.6 & 142.5 & 25.1 & 77.9 & 5.4 \\
\hline Credit to the private sector & 34.8 & 44.6 & -3.8 & 22.3 & 48.8 & 34.8 \\
\hline Credit to the private sector (in percent of non-oil GDP excluding oil derivatives) & 17.4 & 19.9 & 16.8 & 17.8 & 22.1 & 23.7 \\
\hline Broad money (in percent of overall GDP) & 5.1 & 6.6 & 9.0 & 8.0 & 7.0 & 7.1 \\
\hline Velocity (overall GDP/end-of-period M2) & 19.7 & 15.2 & 11.1 & 12.5 & 14.2 & 14.1 \\
\hline Velocity (non-oil GDP excluding oil derivatives/end-of-period M2) & 3.2 & 2.7 & 1.9 & 1.7 & 1.5 & 1.7 \\
\hline Reserve money multiplier (M2/RM) & 1.4 & 2.0 & 1.3 & 1.4 & 1.0 & 1.1 \\
\hline Bank reserves-to-M2-deposits ratio ${ }^{1}$ & 0.6 & 0.3 & 0.70 & 0.65 & 0.95 & 0.85 \\
\hline Currency-to-M2-deposits ratio & 0.4 & 0.4 & 0.29 & 0.28 & 0.26 & 0.27 \\
\hline Lending rate & 18.0 & 18.0 & 18.0 & 18.0 & 17.0 & 15.0 \\
\hline Deposit rate & 5.0 & 5.0 & 5.0 & 5.0 & 4.75 & 4.25 \\
\hline
\end{tabular}

Sources: BEAC and IMF staff estimates and projections.

${ }^{1}$ Government deposits at commercial banks are not part of M2-deposits, but are subject to reserve requirements, which is why this ratio exhibits an upward trend. 
Table 13. Equatorial Guinea: Central Bank and Commercial Banks, 2001-06 (Billions of CFA francs, unless otherwise specified; end of period)

\begin{tabular}{|c|c|c|c|c|c|c|}
\hline & $\begin{array}{l}2001 \\
\text { Dec. }\end{array}$ & $\begin{array}{l}2002 \\
\text { Dec. }\end{array}$ & $\begin{array}{l}2003 \\
\text { Dec. }\end{array}$ & $\begin{array}{l}2004 \\
\text { Dec. }\end{array}$ & $\begin{array}{l}2005 \\
\text { Dec. }\end{array}$ & $\begin{array}{l}2006 \\
\text { Dec. }\end{array}$ \\
\hline \multicolumn{7}{|l|}{ Central Bank } \\
\hline $\begin{array}{l}\text { Net foreign assets } \\
\text { (millions of U.S. dollars) }\end{array}$ & $\begin{array}{l}45.9 \\
62.5\end{array}$ & $\begin{array}{l}50.0 \\
77.6\end{array}$ & $\begin{array}{l}118.0 \\
221.1\end{array}$ & $\begin{array}{l}450.3 \\
920.3\end{array}$ & $\begin{array}{l}1,164.2 \\
2,104.2\end{array}$ & $\begin{array}{l}1,523.0 \\
3,057.9\end{array}$ \\
\hline $\begin{array}{l}\text { Net domestic assets } \\
\text { Claims on government (net) } \\
\text { Claims on commercial banks (net) } \\
\text { Claims on rest of the economy } \\
\text { Other items (net) }\end{array}$ & $\begin{array}{r}1.5 \\
-3.5 \\
0.0 \\
0.0 \\
5.0\end{array}$ & $\begin{array}{r}-0.4 \\
-4.1 \\
0.0 \\
0.0 \\
3.8\end{array}$ & $\begin{array}{r}2.3 \\
-2.6 \\
0.0 \\
0.0 \\
4.9\end{array}$ & $\begin{array}{r}-299.7 \\
-302.4 \\
0.0 \\
0.0 \\
2.6\end{array}$ & $\begin{array}{r}-896.5 \\
-899.2 \\
0.0 \\
0.0 \\
2.7\end{array}$ & $\begin{array}{r}-1,240.8 \\
-1,243.0 \\
0.0 \\
0.0 \\
2.3\end{array}$ \\
\hline $\begin{array}{l}\text { Reserve money } \\
\text { Currency outside banks } \\
\text { Bank reserves } \\
\text { Cash } \\
\text { Deposits } \\
\text { Nonbank deposits }\end{array}$ & $\begin{array}{r}47.4 \\
17.6 \\
29.0 \\
3.9 \\
25.1 \\
0.8\end{array}$ & $\begin{array}{r}49.6 \\
26.0 \\
22.9 \\
6.5 \\
16.4 \\
0.8\end{array}$ & $\begin{array}{r}120.3 \\
35.1 \\
84.4 \\
5.1 \\
79.3 \\
0.8\end{array}$ & $\begin{array}{r}150.5 \\
45.7 \\
104.7 \\
7.3 \\
97.4 \\
0.1\end{array}$ & $\begin{array}{r}267.8 \\
57.7 \\
210.0 \\
9.2 \\
200.8 \\
0.1\end{array}$ & $\begin{array}{r}282.3 \\
68.2 \\
213.4 \\
11.0 \\
202.3 \\
0.7\end{array}$ \\
\hline \multicolumn{7}{|l|}{ Deposit money banks } \\
\hline Net foreign assets & 14.6 & 54.6 & 35.6 & 49.3 & 76.8 & 50.9 \\
\hline $\begin{array}{l}\text { Net domestic assets } \\
\text { Reserves } \\
\text { Cash } \\
\text { Deposits with Central Bank }\end{array}$ & $\begin{array}{r}31.7 \\
29.0 \\
3.9 \\
25.1\end{array}$ & $\begin{array}{r}17.7 \\
22.9 \\
6.5 \\
16.4\end{array}$ & $\begin{array}{r}83.7 \\
84.4 \\
5.1 \\
79.3\end{array}$ & $\begin{array}{r}112.1 \\
104.7 \\
7.3 \\
97.4\end{array}$ & $\begin{array}{r}144.5 \\
210.0 \\
9.2 \\
200.8\end{array}$ & $\begin{array}{r}198.7 \\
213.4 \\
11.0 \\
202.3\end{array}$ \\
\hline $\begin{array}{l}\text { Domestic credit } \\
\text { Claims on Central Bank (net) }\end{array}$ & $\begin{array}{r}12.4 \\
0.0\end{array}$ & $\begin{array}{l}7.5 \\
0.0\end{array}$ & $\begin{array}{r}19.9 \\
0.0\end{array}$ & $\begin{array}{r}33.2 \\
0.0\end{array}$ & $\begin{array}{r}-34.4 \\
0.0\end{array}$ & $\begin{array}{r}18.4 \\
0.0\end{array}$ \\
\hline Claims on government (net) & -24.5 & -46.7 & -31.8 & -29.6 & -128.3 & -111.0 \\
\hline Claims & 0.3 & 4.1 & 2.1 & 5.8 & 3.5 & 3.7 \\
\hline Deposits & -24.8 & -50.8 & -33.8 & -35.4 & -131.8 & -114.8 \\
\hline Claims on nongovernment & 36.9 & 54.2 & 51.6 & 62.8 & 93.8 & 129.5 \\
\hline Public enterprises & 0.4 & 1.5 & 0.9 & 0.8 & 1.4 & 4.9 \\
\hline Private sector & 36.5 & 52.8 & 50.8 & 62.1 & 92.4 & 124.5 \\
\hline Other items (net) & -9.7 & -12.7 & -20.5 & -25.9 & -31.0 & -33.1 \\
\hline Deposit liabilities to nonbank residents & 47.1 & 73.1 & 120.1 & 161.4 & 221.4 & 250.3 \\
\hline
\end{tabular}

Sources: Data provided by Equatoguinean authorities, and IMF staff estimates and projections. 
Table 14. Equatorial Guinea: Balance of Payments, 2001-06 ${ }^{1}$

(Millions of U.S. dollars, unless otherwise specified)

\begin{tabular}{|c|c|c|c|c|c|c|}
\hline & 2001 & 2002 & 2003 & $\begin{array}{r}2004 \\
\text { Est. }\end{array}$ & $\begin{array}{r}2005 \\
\text { Est. }\end{array}$ & $\begin{array}{r}2006 \\
\text { Est. }\end{array}$ \\
\hline Current account & -709.5 & 1.6 & -987.6 & $-1,072.6$ & -417.3 & 377.6 \\
\hline $\begin{array}{l}\text { Trade balance } \\
\text { Exports of goods, f.o.b. }\end{array}$ & $\begin{array}{r}929.7 \\
1,738.4\end{array}$ & $\begin{array}{l}1,605.8 \\
2,113.2\end{array}$ & $\begin{array}{l}1,588.0 \\
2,825.1\end{array}$ & $\begin{array}{l}3,135.5 \\
4,715.4\end{array}$ & $\begin{array}{l}5,091.3 \\
7,214.7\end{array}$ & $\begin{array}{l}5,391.5 \\
8,013.5\end{array}$ \\
\hline Of which: Hydrocarbons exports & $1,672.7$ & $2,049.2$ & $2,750.3$ & $4,633.9$ & $7,141.3$ & $7,931.3$ \\
\hline Of which: Crude oil & $1,569.3$ & $1,909.0$ & $2,557.9$ & $4,342.2$ & $6,563.5$ & $7,197.3$ \\
\hline $\begin{array}{l}\text { Liquefied natural gas } \\
\text { Liquefied petroleum gas }\end{array}$ & $\begin{array}{r}0.0 \\
18.7\end{array}$ & $\begin{array}{r}0.0 \\
18.2\end{array}$ & $\begin{array}{r}0.0 \\
23.2\end{array}$ & $\begin{array}{r}0.0 \\
28.9\end{array}$ & $\begin{array}{r}0.0 \\
188.7\end{array}$ & $\begin{array}{r}0.0 \\
410.3\end{array}$ \\
\hline Methanol & 84.7 & 121.9 & 169.3 & 262.8 & 389.1 & 323.7 \\
\hline Timber & 52.9 & 47.7 & 53.6 & 56.3 & 50.3 & 57.1 \\
\hline Cocoa & 1.6 & 2.3 & 4.4 & 6.1 & 3.5 & 4.7 \\
\hline Coffee & 0.0 & 0.0 & 0.1 & 0.1 & 0.1 & 0.1 \\
\hline Imports of goods, f.o.b. & -808.7 & -507.4 & $-1,237.2$ & $-1,579.9$ & $-2,123.4$ & $-2,622.0$ \\
\hline Of which: Petroleum sector & -665.0 & -305.8 & -928.5 & -943.7 & $-1,175.4$ & $-1,260.0$ \\
\hline Petroleum products & -27.3 & -27.8 & -37.9 & -62.4 & -95.1 & -114.3 \\
\hline Public sector equipment & -89.4 & -127.2 & -204.4 & -481.0 & -697.4 & $-1,053.7$ \\
\hline Other $^{2}$ & -27.0 & -46.5 & -66.3 & -92.7 & -155.5 & -193.9 \\
\hline Services & -768.8 & -590.9 & -985.0 & $-1,251.1$ & $-1,393.4$ & $-1,593.5$ \\
\hline Credit & 21.3 & 25.4 & 34.2 & 50.9 & 70.3 & 82.9 \\
\hline Debit & -790.1 & -616.3 & $-1,019.2$ & $-1,302.0$ & $-1,463.8$ & $-1,676.5$ \\
\hline Income & -868.3 & $-1,002.2$ & $-1,564.3$ & $-2,911.1$ & $-4,048.4$ & $-3,328.0$ \\
\hline Investment income (net) & -824.9 & -948.8 & $-1,491.7$ & $-2,791.3$ & $-3,866.0$ & $-3,136.4$ \\
\hline Credit & 2.5 & 11.9 & 20.1 & 28.4 & 29.3 & 41.8 \\
\hline Debit $^{3}$ & -827.3 & -960.7 & $-1,511.8$ & $-2,819.7$ & $-3,895.3$ & $-3,178.3$ \\
\hline Current transfers & -2.1 & -11.1 & -26.2 & -45.9 & -66.7 & -92.4 \\
\hline Public & 14.4 & 15.1 & 18.2 & 20.0 & 20.0 & 20.2 \\
\hline Private & -16.5 & -26.2 & -44.4 & -65.9 & -86.8 & -112.6 \\
\hline Capital and financial account & 743.9 & -92.0 & $1,140.1$ & $1,738.3$ & $1,846.8$ & 357.1 \\
\hline Capital account & 6.4 & 0.0 & 0.0 & 0.0 & 0.0 & 0.0 \\
\hline Financial account & 737.4 & -92.0 & $1,140.1$ & $1,738.3$ & $1,846.8$ & 357.1 \\
\hline Direct investment & 940.8 & 323.4 & $1,443.5$ & $1,651.8$ & $1,871.5$ & $1,861.4$ \\
\hline Direct investment abroad & -4.2 & 0.0 & 0.0 & 0.0 & 0.0 & 0.0 \\
\hline Direct investment to Equatorial Guinea & 945.0 & 323.4 & $1,443.5$ & $1,651.8$ & $1,871.5$ & $1,861.4$ \\
\hline Portfolio investment (net) & -0.4 & 0.0 & 0.0 & 0.0 & 0.0 & 0.0 \\
\hline Other investment (net) & -203.0 & -415.4 & -303.4 & 86.5 & -24.7 & $-1,504.3$ \\
\hline Medium- and long-term transactions & -7.8 & -7.7 & -18.6 & -14.2 & -45.2 & -101.2 \\
\hline General government & -7.8 & -7.5 & -7.0 & -8.3 & -36.3 & -95.3 \\
\hline Incl.: Disbursements & 0.0 & 0.0 & 0.0 & 0.0 & 0.0 & 0.0 \\
\hline Amortization & -7.8 & -7.5 & -7.0 & -8.3 & -36.3 & -95.3 \\
\hline Banks & 0.0 & 0.0 & 0.0 & 0.0 & 0.0 & 0.0 \\
\hline Other sectors & 0.0 & -0.2 & -11.5 & -5.9 & -9.0 & -5.9 \\
\hline Short-term transactions & -195.2 & -407.7 & -284.8 & 100.7 & 20.6 & $-1,403.0$ \\
\hline General government ${ }^{4}$ & -116.5 & -287.4 & -178.2 & -43.7 & -105.7 & $-1,289.8$ \\
\hline Banks & -3.8 & -61.5 & 32.7 & -25.9 & -52.1 & 49.5 \\
\hline Other sectors & -74.9 & -58.8 & -139.4 & 170.4 & 178.4 & -162.7 \\
\hline Errors and omissions & 40.6 & 97.8 & -12.2 & 16.1 & 16.7 & -23.2 \\
\hline Overall balance & 75.0 & 7.4 & 140.3 & 681.8 & $1,446.2$ & 711.5 \\
\hline Financing & -75.0 & -7.4 & -140.3 & -681.8 & $-1,446.2$ & -711.5 \\
\hline Change in net international reserves ${ }^{5}$ (increase -) & -76.3 & -8.0 & -134.9 & -658.9 & $-1,415.8$ & -711.5 \\
\hline Exceptional financing & 1.3 & 0.6 & -5.3 & -22.9 & -30.3 & 0.0 \\
\hline Change in arrears (net; decrease -) & -10.2 & 0.6 & -75.2 & -22.9 & -85.2 & 0.0 \\
\hline Debt relief & 11.4 & 0.0 & 69.9 & 0.0 & 54.9 & 0.0 \\
\hline \multicolumn{7}{|l|}{ Memorandum items: } \\
\hline Gross official foreign assets (USD million) & 265.5 & 567.1 & 890.6 & $1,633.1$ & 2,921.6 & $5,175.0$ \\
\hline Of which: Reserve assets at the BEAC & 71.7 & 86.0 & 231.3 & 930.1 & $2,112.9$ & $3,076.4$ \\
\hline Government bank deposits abroad & 193.8 & 481.1 & 659.3 & 703.0 & 808.7 & $2,098.6$ \\
\hline Gross official foreign assets (months of next year's imports) & 2.8 & 3.0 & 3.7 & 5.5 & 8.2 & 12.0 \\
\hline Gross official foreign assets (months of next year's imports (excl. petroleum sector)) & 9.6 & 11.5 & 10.6 & 13.6 & 17.8 & 23.9 \\
\hline Current account balance (percent of GDP; deficit -) & -40.8 & 0.1 & -33.3 & -21.9 & -5.5 & 4.4 \\
\hline Growth of hydrocarbons exports (percent) & 47.3 & 22.5 & 34.2 & 68.5 & 54.1 & 11.1 \\
\hline Growth of non-oil exports (percent) & -20.7 & -2.6 & 16.9 & 8.9 & -9.9 & 12.1 \\
\hline Growth of other imports & & & 20.3 & 13.4 & 3.3 & 3.8 \\
\hline
\end{tabular}

Sources: Equatoguinean authorities, and IMF staff estimates and projections.

${ }^{1}$ The BOP data presented in this table are not compiled in accordance with the IMF's Balance of Payments Manual, fifth edition. At least since 2003, the data have not been derived from customs' and bank records' data, but from estimates by the national office of the BEAC, which are based on a financial program. Fund staff have made ad hoc adjustments to both historical and forecasted data.

${ }^{2}$ Including private sector consumption and investment imports.

${ }^{3}$ Including investment income of oil companies. The latter includes reinvested earnings (with an offsetting entry in foreign direct investment).

${ }^{4}$ Since 2000 , entries represent changes in government deposits in commercial

${ }^{5}$ Consists only of items on the balance sheet of the national office of the BEAC (i.e., excluding government bank deposits abroad). 
Table 15. Equatorial Guinea: Composition of Imports, 2001-06

\begin{tabular}{lrrrrrr}
\hline & 2001 & 2002 & 2003 & 2004 & 2005 & 2006 \\
\hline & \multicolumn{7}{c}{ (Millions of U.S. dollars) } \\
Total imports, f.o.b. & 809 & 507 & 1,237 & 1,580 & 2,123 & 2,622 \\
Public sector equipment & 89 & 127 & 204 & 481 & 697 & 1,054 \\
Petroleum sector & 665 & 306 & 929 & 944 & 1,175 & 1,260 \\
Petroleum products & 27 & 28 & 38 & 62 & 95 & 114 \\
Other & 27 & 47 & 66 & 93 & 156 & 194 \\
& & & & & & \\
& & & & & & \\
Total imports, f.o.b. & 100.0 & 100.0 & 100.0 & 100.0 & 100.0 & 100.0 \\
Public sector & 11.1 & 25.1 & 16.5 & 30.4 & 32.8 & 40.2 \\
Oil sector & 82.2 & 60.3 & 75.1 & 59.7 & 55.4 & 48.1 \\
Petroleum products & 3.4 & 5.5 & 3.1 & 4.0 & 4.5 & 4.4 \\
Other & 3.3 & 9.2 & 5.4 & 5.9 & 7.3 & 7.4 \\
\hline
\end{tabular}

Sources: Equatoguinean authorities and IMF staff estimates.

Table 16. Equatorial Guinea: Direction of Trade, 2001-06

\begin{tabular}{|c|c|c|c|c|c|c|}
\hline & 2001 & 2002 & 2003 & 2004 & 2005 & 2006 \\
\hline Total exports & 100.0 & 100.0 & 100.0 & 100.0 & 100.0 & 100.0 \\
\hline United States & 27.8 & 29.0 & 33.2 & 29.1 & 24.6 & $\ldots$ \\
\hline Canada & 4.5 & 10.8 & 12.7 & 6.8 & 7.3 & $\ldots$ \\
\hline Japan & 0.9 & 2.6 & 0.0 & 1.1 & 3.3 & $\ldots$ \\
\hline France & 2.3 & 5.1 & 3.6 & 2.1 & 4.0 & $\ldots$ \\
\hline Germany & 0.3 & 0.3 & 0.1 & 0.2 & 0.5 & $\ldots$ \\
\hline Italy & 0.0 & 1.4 & 6.3 & 3.9 & 3.6 & $\ldots$ \\
\hline Netherlands & 2.0 & 1.0 & 1.9 & 1.3 & 5.2 & $\ldots$ \\
\hline Spain & 34.4 & 25.9 & 25.4 & 15.9 & 10.8 & $\ldots$ \\
\hline United Kingdom & 0.5 & 0.5 & 0.4 & 0.5 & 0.4 & $\ldots$ \\
\hline China & 21.7 & 17.9 & 14.2 & 22.6 & 21.8 & $\ldots$ \\
\hline Brazil & 0.7 & 0.2 & 0.0 & 0.3 & 4.6 & $\ldots$ \\
\hline Other & 2.6 & 5.1 & 2.2 & 16.2 & 13.7 & $\ldots$ \\
\hline Total imports & 100.0 & 100.0 & 100.0 & 100.0 & 100.0 & 100.0 \\
\hline United States & 27.8 & 27.0 & 30.2 & 32.1 & 24.5 & $\ldots$ \\
\hline Canada & 0.7 & 0.3 & 0.2 & 0.2 & 0.2 & $\ldots$ \\
\hline Japan & 0.3 & 0.4 & 0.3 & 1.2 & 0.6 & $\ldots$ \\
\hline France & 8.7 & 9.6 & 14.9 & 10.5 & 12.1 & $\ldots$ \\
\hline Germany & 1.6 & 1.3 & 0.7 & 0.8 & 0.8 & $\ldots$ \\
\hline Italy & 8.1 & 4.4 & 5.2 & 5.2 & 20.6 & $\ldots$ \\
\hline Netherlands & 4.2 & 4.4 & 1.9 & 3.5 & 2.4 & $\ldots$ \\
\hline Spain & 15.4 & 14.8 & 8.1 & 16.5 & 10.8 & $\ldots$ \\
\hline United Kingdom & 12.4 & 13.7 & 15.8 & 9.4 & 6.9 & $\ldots$ \\
\hline China & 0.9 & 0.7 & 0.5 & 1.5 & 1.9 & $\ldots$ \\
\hline Côte d'Ivoire & 4.7 & 4.4 & 12.1 & 5.4 & 8.6 & $\ldots$ \\
\hline Others & 10.7 & 16.5 & 8.8 & 11.2 & 8.9 & $\ldots$ \\
\hline
\end{tabular}

Sources: IMF, Direction of Trade Statistics Yearbook. 
Table 17. Equatorial Guinea: Scheduled External Public Debt Service, 2001-06

(Millions of U.S. dollars, unless otherwise indicated)

\begin{tabular}{|c|c|c|c|c|c|c|}
\hline & 2001 & 2002 & 2003 & 2004 & 2005 & 2006 \\
\hline Multilateral creditors & 6.3 & 2.8 & 5.1 & 4.8 & 4.0 & 4.0 \\
\hline Principal & 4.6 & 2.0 & 3.9 & 3.5 & 2.8 & 3.1 \\
\hline Interest & 1.7 & 0.8 & 1.2 & 1.3 & 1.2 & 0.9 \\
\hline Bilateral creditors & 6.1 & 3.5 & 4.6 & 6.1 & 34.7 & 97.1 \\
\hline Principal & 2.7 & 1.3 & 3.1 & 4.9 & 33.6 & 96.4 \\
\hline Interest & 3.4 & 2.2 & 1.5 & 1.2 & 1.1 & 0.7 \\
\hline Paris Club creditors & 5.8 & 3.5 & 3.0 & 2.9 & 31.4 & 91.4 \\
\hline Principal & 2.5 & 1.3 & 1.5 & 1.7 & 30.3 & 90.7 \\
\hline Interest & 3.3 & 2.2 & 1.5 & 1.2 & 1.1 & 0.7 \\
\hline Other bilateral creditors & 0.3 & 0.0 & 1.6 & 3.2 & 3.3 & 5.7 \\
\hline Principal & 0.2 & 0.0 & 1.6 & 3.2 & 3.3 & 5.7 \\
\hline Interest & 0.1 & 0.0 & 0.0 & 0.0 & 0.0 & 0.0 \\
\hline Total & 18.5 & 9.8 & 9.8 & 10.9 & 38.7 & 101.0 \\
\hline Principal & 10.0 & 4.5 & 7.0 & 8.3 & 36.4 & 99.4 \\
\hline Interest & 8.5 & 5.3 & 2.7 & 2.5 & 2.3 & 1.6 \\
\hline \multicolumn{7}{|l|}{ Memorandum items: } \\
\hline \multicolumn{7}{|l|}{ Debt-service ratio } \\
\hline Percent of exports of goods and nonfactor services & 1.1 & 0.5 & 0.3 & 0.2 & 0.5 & 1.2 \\
\hline Percent of government revenue & 3.9 & 1.7 & 1.2 & 0.7 & 1.4 & 2.6 \\
\hline
\end{tabular}

Sources: Equatoguinean authorities and IMF staff estimates.

Table 18. Equatorial Guinea: External Medium- and Long-Term Outstanding Public Debt, 2001-06 (Millions of U.S. Dollars, unless otherwise specified)

\begin{tabular}{|c|c|c|c|c|c|c|}
\hline & 2001 & 2002 & 2003 & 2004 & 2005 & 2006 \\
\hline Total outstanding debt & 239.9 & 212.3 & 299.2 & 318.0 & 246.6 & 156.8 \\
\hline Multilateral debt & 89.1 & 91.3 & 108.5 & 113.7 & 97.8 & 105.8 \\
\hline African Development Bank/African Development Fund & 30.1 & 32.0 & 42.8 & 45.0 & 39.2 & 43.0 \\
\hline IDA & 45.0 & 44.6 & 51.1 & 54.9 & 47.6 & 51.6 \\
\hline IMF & 2.5 & 1.1 & 1.0 & 0.0 & 0.0 & 0.0 \\
\hline Other & 11.5 & 13.6 & 13.5 & 13.7 & 11.1 & 11.1 \\
\hline Bilateral debt & 150.8 & 121.0 & 189.5 & 202.9 & 147.6 & 49.7 \\
\hline Paris Club creditors & 88.5 & 83.3 & 151.8 & 163.8 & 115.9 & 33.8 \\
\hline Non-Paris Club creditors & 62.4 & 37.8 & 37.7 & 39.2 & 31.7 & 15.9 \\
\hline
\end{tabular}

Sources: Data provided by Equatoguinean authorities and IMF staff estimates. 
Table 19. Equatorial Guinea: Exchange Rates, 2001-06

\begin{tabular}{|c|c|c|c|c|c|c|c|c|}
\hline & \multicolumn{2}{|c|}{$\begin{array}{l}\text { Nominal Effective } \\
\text { Exchange Rate }\end{array}$} & \multicolumn{2}{|c|}{$\begin{array}{l}\text { Real Effective } \\
\text { Exchange Rate }\end{array}$} & \multicolumn{2}{|c|}{ CFA franc per U.S. dollar } & \multicolumn{2}{|c|}{ CFA franc per SDR } \\
\hline & Index $^{1}$ & $\begin{array}{c}\text { Annual } \\
\text { percentage } \\
\text { change }\end{array}$ & Index $^{1}$ & $\begin{array}{c}\text { Annual } \\
\text { percentage } \\
\text { change }\end{array}$ & $\begin{array}{l}\text { Period } \\
\text { average }\end{array}$ & $\begin{array}{l}\text { End of } \\
\text { period }\end{array}$ & $\begin{array}{l}\text { Period } \\
\text { average }\end{array}$ & $\begin{array}{l}\text { End of } \\
\text { period }\end{array}$ \\
\hline \multicolumn{9}{|l|}{ Annual } \\
\hline 2001 & 99.0 & -1.0 & 105.1 & 5.1 & 733.0 & 744.3 & 932.4 & 935.4 \\
\hline 2002 & 102.3 & 3.3 & 114.9 & 9.3 & 697.0 & 625.5 & 899.3 & 850.4 \\
\hline 2003 & 114.0 & 11.4 & 134.4 & 16.9 & 581.2 & 519.4 & 811.4 & 771.8 \\
\hline 2004 & 119.8 & 5.1 & 143.8 & 7.0 & 528.3 & 481.6 & 781.9 & 747.9 \\
\hline 2005 & 119.6 & -0.1 & 147.7 & 2.7 & 527.5 & 556.0 & 777.9 & 794.7 \\
\hline 2006 & 120.2 & 0.4 & 151.2 & 2.4 & 522.9 & 498.1 & 768.7 & 749.3 \\
\hline
\end{tabular}

Sources: IMF, International Finance Statistics; IMF, Information Notice System.

${ }^{1} 2000=100$. 


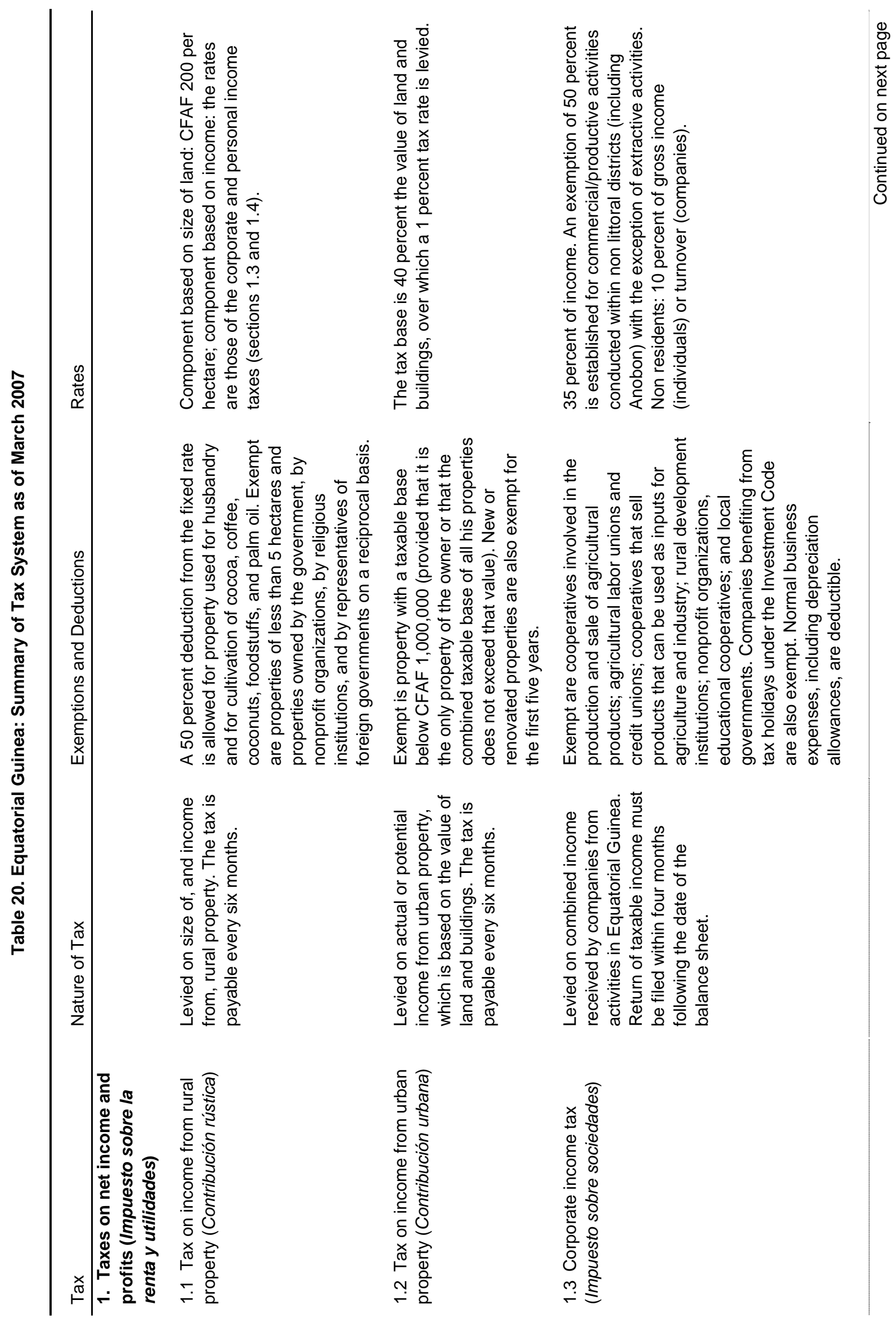




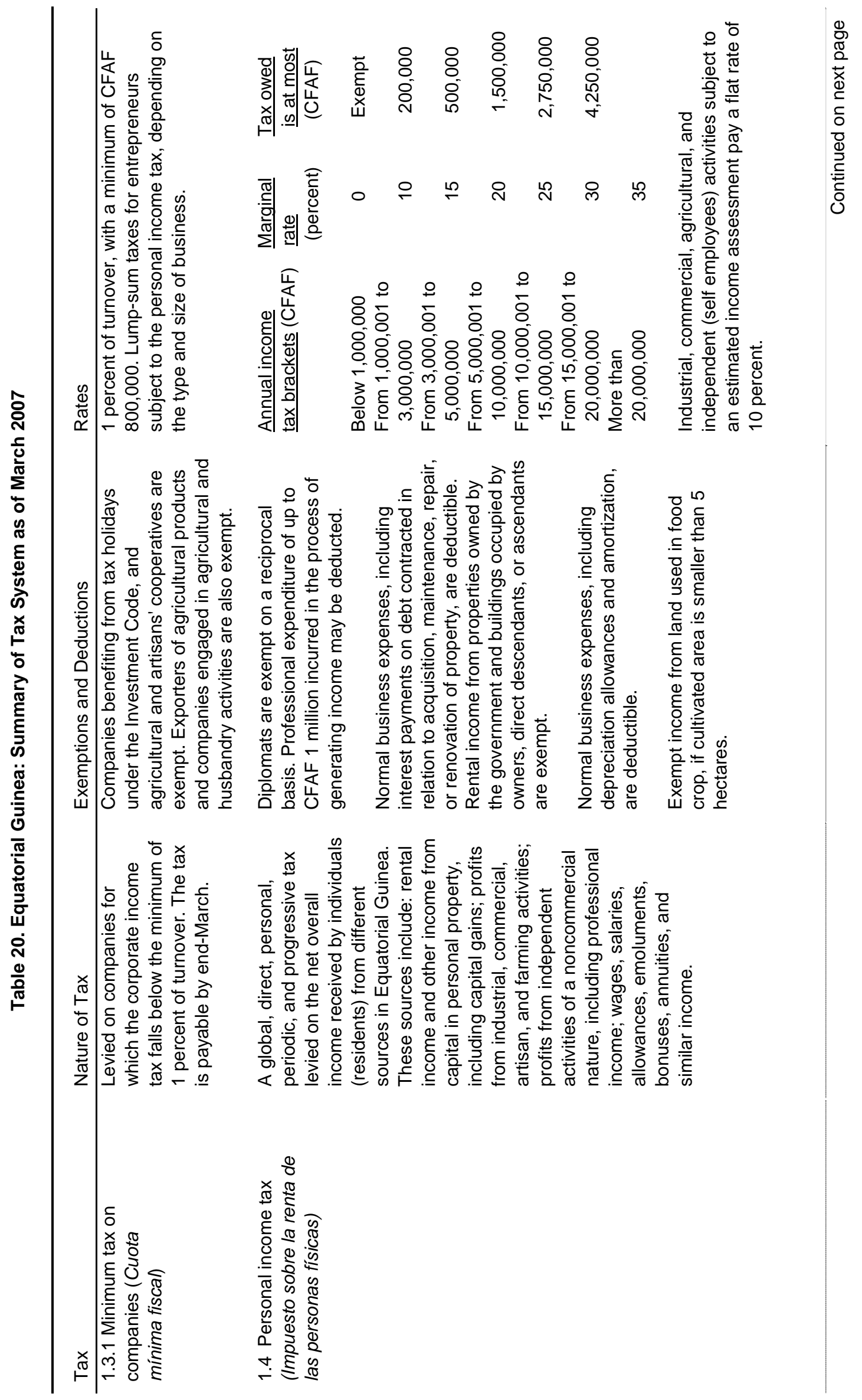




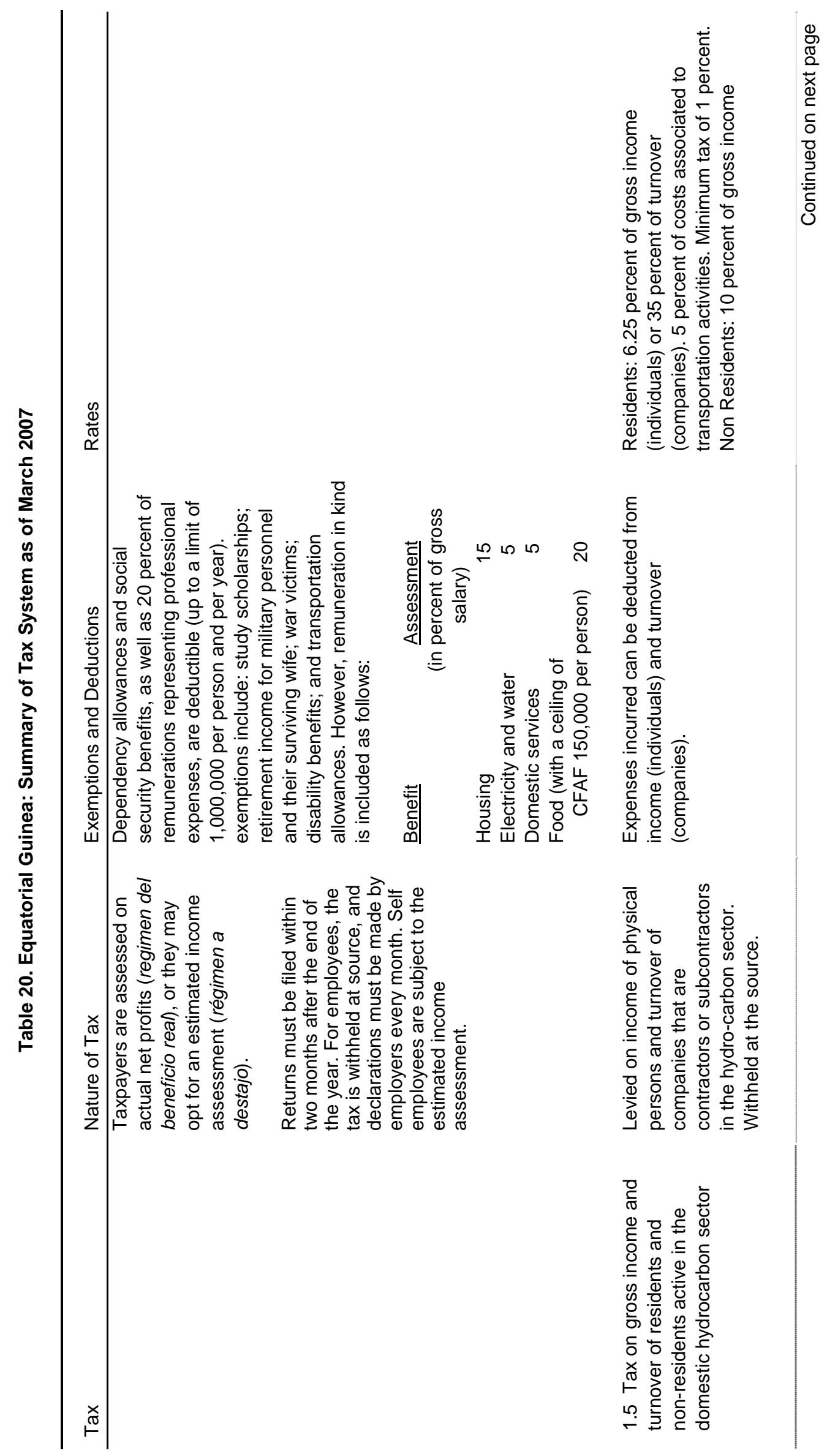




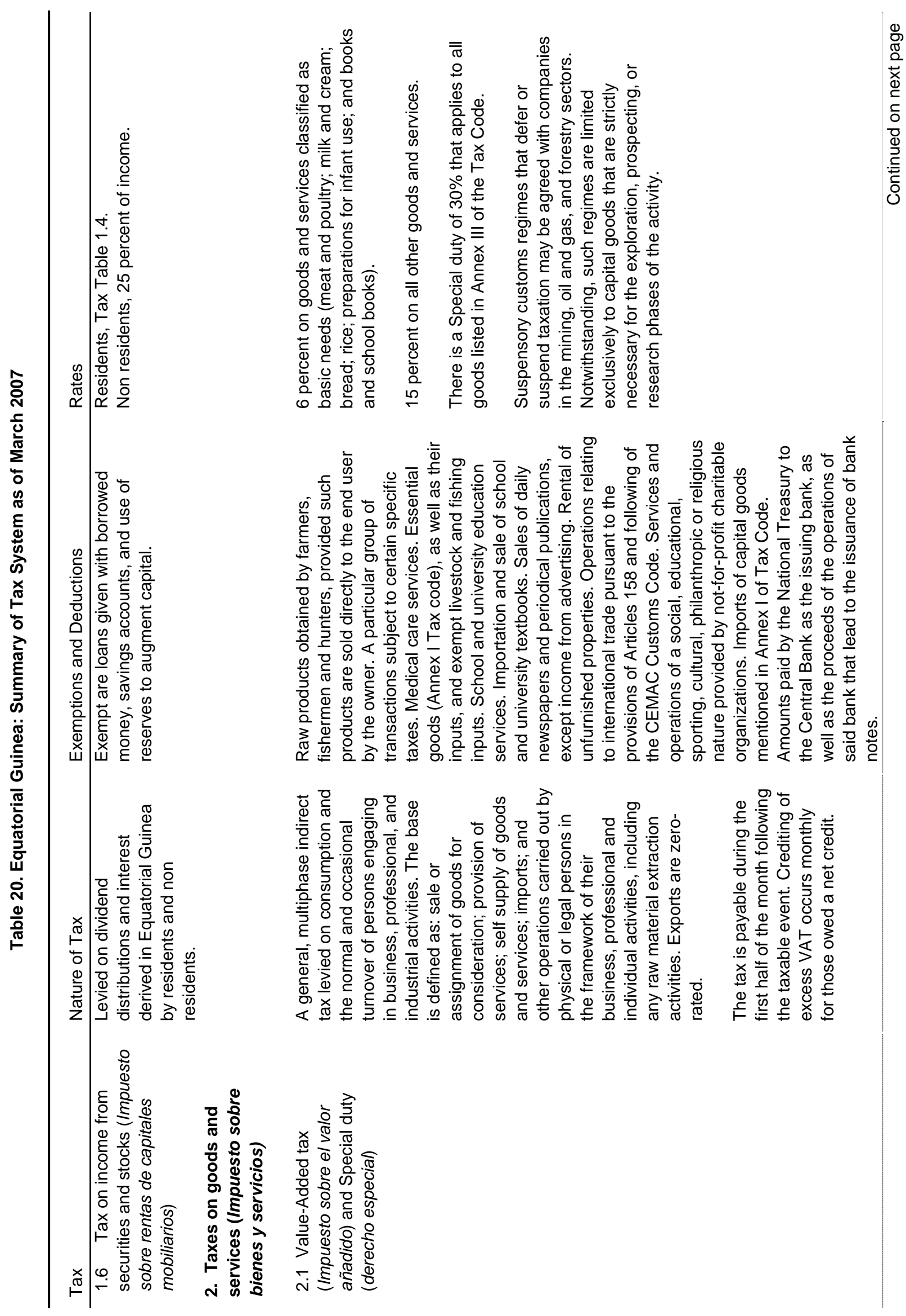




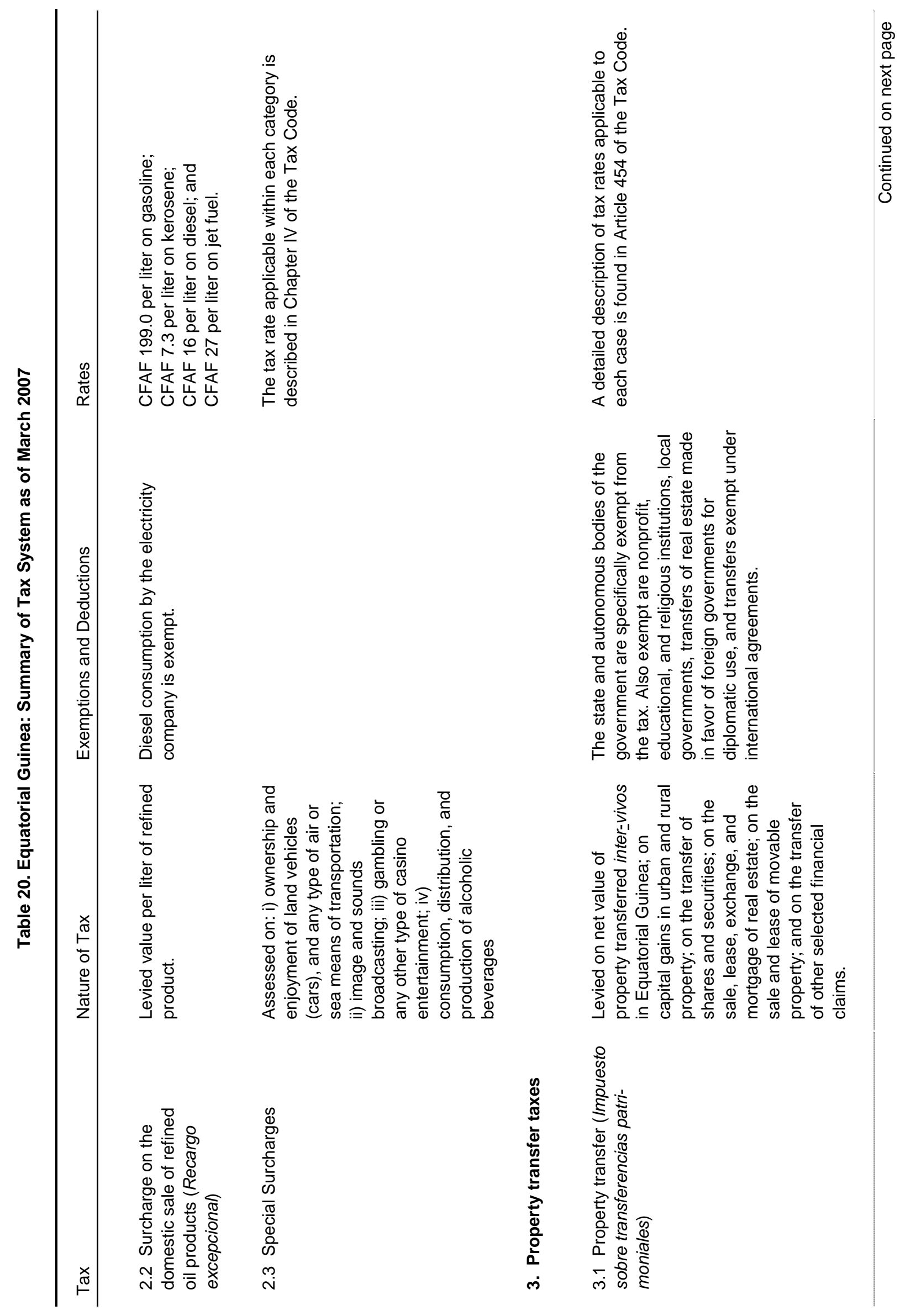




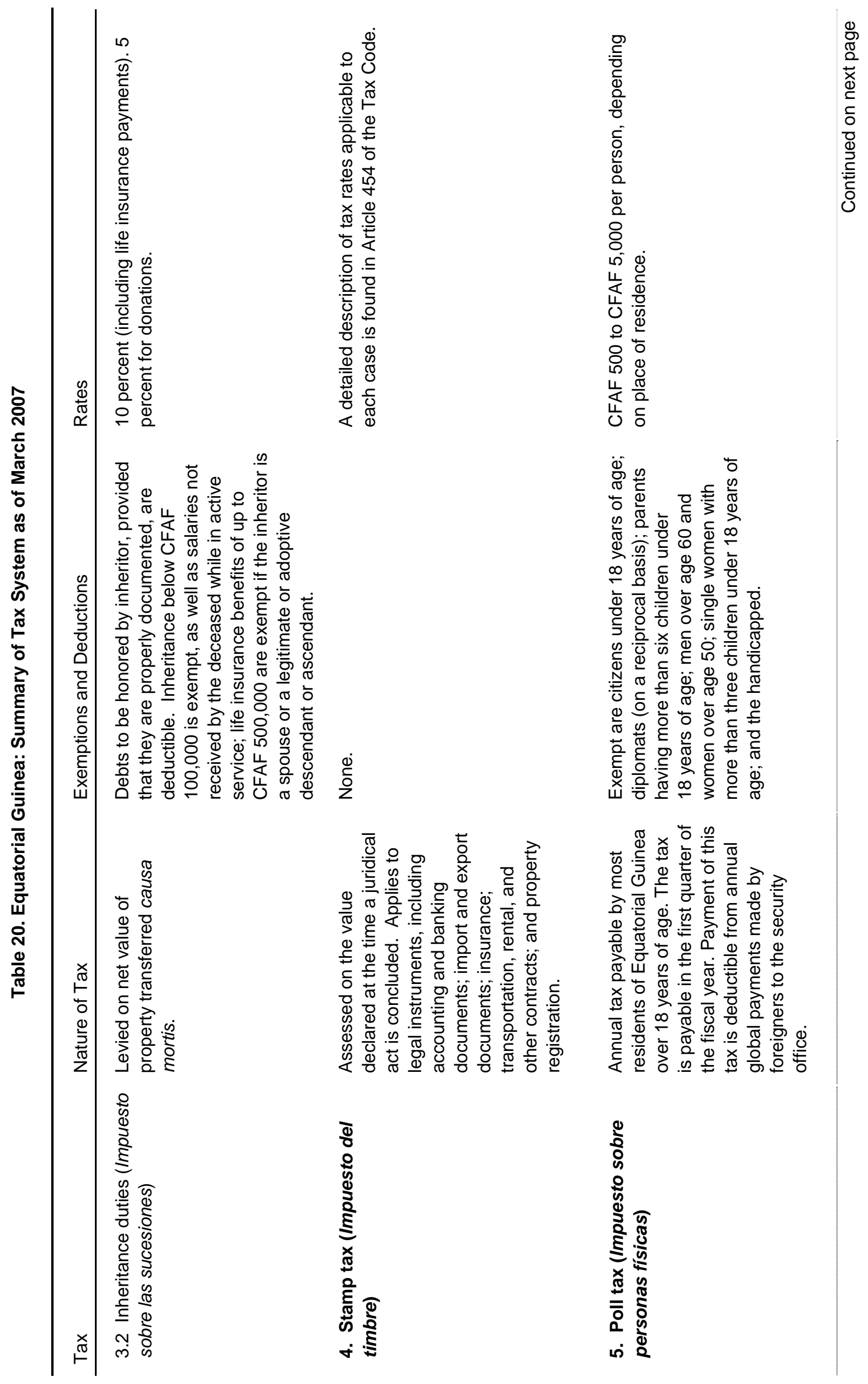




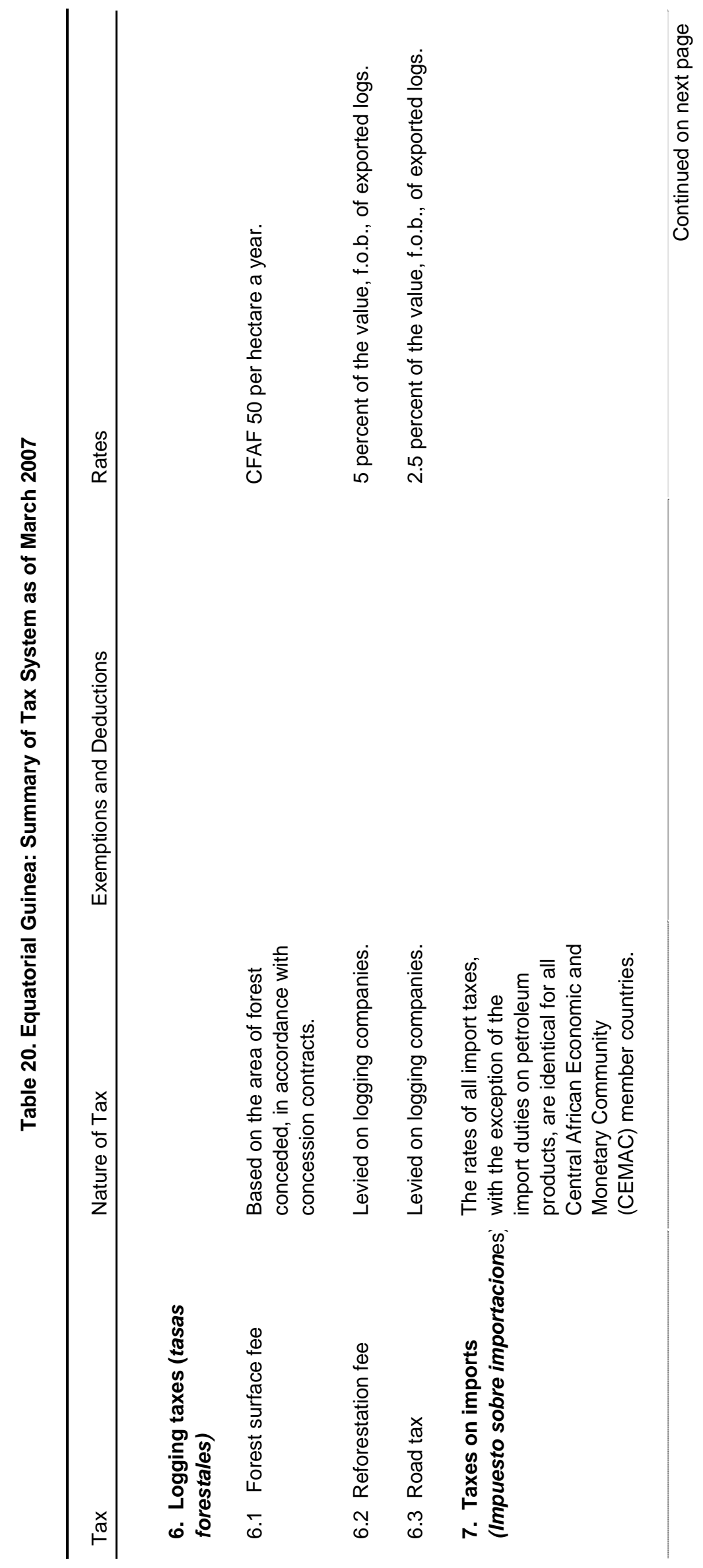




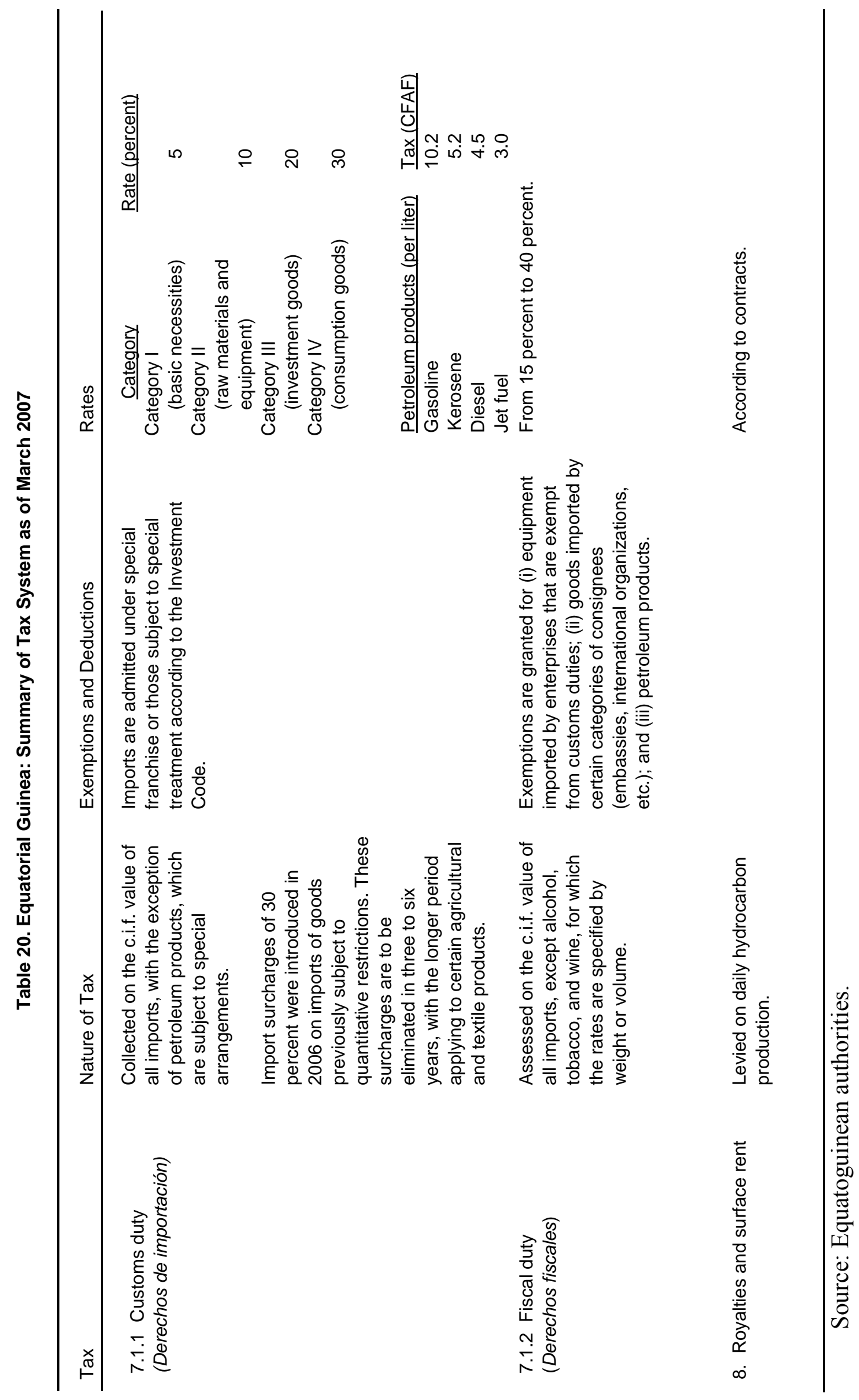

